\title{
DSCAM Deficiency Causes Loss of Pre-Inspiratory Neuron Synchroneity and Perinatal Death
}

\author{
Kenji Amano, ${ }^{1}$ Morimitsu Fujii, ${ }^{2}$ Satoru Arata, ${ }^{7}$ Takuro Tojima, ${ }^{3}$ Masaharu Ogawa, ${ }^{4}$ Noriyuki Morita, ${ }^{5}$ \\ Atsushi Shimohata, ${ }^{1}$ Teiichi Furuichi, ${ }^{5}$ Shigeyoshi Itohara, ${ }^{6}$ Hiroyuki Kamiguchi, ${ }^{3}$ Julie R. Korenberg, ${ }^{8}$ Akiko Arata, ${ }^{2,9}$ \\ and Kazuhiro Yamakawa ${ }^{1}$ \\ Laboratories for ${ }^{1}$ Neurogenetics, ${ }^{2}$ Memory and Learning, ${ }^{3}$ Neuronal Growth Mechanisms, ${ }^{4}$ Cell Culture Development, ${ }^{5}$ Molecular Neurogenesis, and \\ ${ }^{6}$ Behavioral Genetics, RIKEN Brain Science Institute, Wako-shi, Saitama 351-0198, Japan, ${ }^{7}$ Center for Biotechnology, Showa University, Shinagawa-ku, \\ Tokyo 142-8555, Japan, ${ }^{8}$ The Brain Institute, University of Utah, Salt Lake City, Utah 84108, and ${ }^{9}$ Division of Physiome, Department of Physiology, Hyogo \\ College of Medicine, Nishinomiya, Hyogo 663-8501, Japan
}

Down syndrome cell adhesion molecule (DSCAM) is a neural adhesion molecule that plays diverse roles in neural development. We disrupted the Dscam locus in mice and found that the null mutants $\left(D s c a m^{-1-}\right)$ died within $24 \mathrm{~h}$ after birth. Whole-body plethysmography showed irregular respiration and lower ventilatory response to hypercapnia in the null mutants. Furthermore, a medulla-spinal cord preparation of $D$ scam ${ }^{-1-}$ mice showed that the C4 ventral root activity, which drives diaphragm contraction for inspiration, had an irregular rhythm with frequent apneas. Optical imaging of the preparation using voltage-sensitive dye revealed that the pre-inspiratory neurons located in the rostral ventrolateral medulla and belonging to the rhythm generator for respiration, lost their synchroneity in Dscam ${ }^{-1-}$ mice. $D$ scam ${ }^{+/-}$mice, which survived to adulthood without any overt abnormalities, also showed irregular respiration but milder than Sscam $^{-1-}$ mice. These results suggest that DSCAM plays a critical role in central respiratory regulation in a dosagedependent manner.

\section{Introduction}

Cell adhesion molecules (CAMs) of the neural Ig superfamily mediate a variety of cell-cell interactions that are important for neural development and function, including neural migration, neurite recognition, fasciculation, and outgrowth. We previously identified the Down syndrome cell adhesion molecule (DSCAM) gene on human chromosome 21q22 and proposed it as an intriguing candidate gene for the etiology of Down syndrome (Yamakawa et al., 1998). It encodes a protein of $2012 \mathrm{aa}$, a member of the largest class of proteins within the neural Ig superfamily (the length corrected in the study by Agarwala et al., 2001). DSCAM mediates cation-independent homophilic binding activity between DSCAM-expressing cells (Agarwala et al., 2000). We found previously a Dscam mouse ortholog that encodes a 2013 aa protein and shows 90 and 98\% identity to its human counterpart at the nucleotide and amino acid levels, respectively (Agarwala et al., 2001). Dscam is widely expressed in the mouse nervous system

Received July 31, 2008; revised Jan. 15, 2009; accepted Jan. 17, 2009.

This work was partly supported by a grant from RIKEN Brain Science Institute, Grant-in-Aid for Scientific Research (KAKENHI) from the Japanese Ministry of Education, Culture, Sports, Science, and Technology, and National Institute of Child Health and Development Grant R01HD45225 (J.R.K.). We thank Dr. J. Miyazaki for providing CAG-cre transgenic mice, Dr. T. Takeuchi, N. Nishiyama, and Y. Onodera for technical support, Dr. K. Yamaguchi for helpful suggestions, and Dr. S. Ganesh for reviewing this manuscript.

Correspondence should be addressed to either of the following: Dr. Kazuhiro Yamakawa, Laboratory for Neurogenetics, RIKEN Brain Science Institute, 2-1 Hirosawa, Wako-shi, Saitama 351-0198, Japan, E-mail: yamakawa@brain.riken.jp; or Dr. Akiko Arata, Division of Physiome, Department of Physiology, Hyogo College of Medicine, 1-1 Mukogawa, Nishinomiya, Hyogo 663-8501, Japan, E-mail: akoarata@hyo-med.ac.jp or ako@brain.riken.jp.

DOI:10.1523/JNEUROSCI.3624-08.2009

Copyright $\odot 2009$ Society for Neuroscience $\quad$ 0270-6474/09/292984-13\$15.00/0 from early embryogenesis through adult. In mouse retina, it has been shown that DSCAM and its self-avoiding activity are required for neurite arborization, mosaic spacing, and prevention of self-fasciculation (Fuerst et al., 2008). Mouse DSCAM has also been reported as a netrin receptor and mediates spinal commissural axon trajectories in collaboration with deleted in colorectal carcinomas (Ly et al., 2008).

DSCAM orthologs were also found in other species, including the fruit fly (Schmucker et al., 2000), zebrafish (Yimlamai et al., 2005), planarian (Fusaoka et al., 2006), and chicken (Yamagata and Sanes, 2008). Dscam was shown to be required for axon guidance in Drosophila, and its null mutants died at larval stages (Schmucker et al., 2000; Wang et al., 2002; Hummel et al., 2003). Alternative splicing of Dscam mRNA in Drosophila potentially generates 38,016 isoforms (Schmucker et al., 2000), although such hyper-alternative splicings have not been detected in human, mouse, chicken, zebrafish, and planarian. Dscam isoforms in Drosophila mediate isoneuronal self-avoidance that underlies dendrite arborization (Hughes et al., 2007; Matthews et al., 2007; Soba et al., 2007). Knockdown of zebrafish Dscam in vivo revealed growth defects that are associated with embryos displaying fewer neurons and misguided axons (Yimlamai et al., 2005). Planarian DSCAM also binds homophilically and plays crucial roles in neuronal cell migration, axon outgrowth, fasciculation, and projection (Fusaoka et al., 2006). In chick retina, DSCAM has been reported to play a role in lamina-specific arborization (Yamagata and Sanes, 2008).

Here we describe that Dscam deficiency in mice under a specific genetic background leads to death shortly after birth with 
central respiratory defects. Physiological analysis revealed that pre-inspiratory (Pre-I) neurons were functionally defective in the $D_{\text {scam }}{ }^{-1-}$ mice. These results indicate that Dscam deficiency in mice leads to specific defects in central respiratory regulation.

\section{Materials and Methods}

Generation of Dscam-null mouse. To obtain genomic clones covering the first exon of the Dscam gene, we screened the mouse PAC library (RPCI21: mouse 129S6/SvEvTAC; Children's Hospital Oakland Research Institute, Oakland, CA) using a $3 \mathrm{~kb}$ fragment (nucleotides 111-2927 of the mouse Dscam cDNA; GenBank accession number NM_031174) as a probe and identified two independent clones (639K11 and 671I13). The genomic structure of a region $(\sim 30 \mathrm{~kb})$ containing the first exon was confirmed by Southern blot analysis with several restriction enzymes and probes in these two clones. A $2.8 \mathrm{~kb}$ blunt-ended EcoRI-SpeI fragment of the $639 \mathrm{~K} 11$ clone was inserted into a blunt-ended SalI site of a targeting vector, generating the $3^{\prime}$ arm of the construct. The $5^{\prime}$ arm was generated by inserting an $8.8 \mathrm{~kb}$ blunt-ended BamHI-EcoRI fragment of the $639 \mathrm{~K} 11$ clone into the blunt-ended $\mathrm{XbaI}$ site, followed by insertion of a loxP fragment into the $M l u \mathrm{I}$ site, thus creating the neomycin phosphotransferase (neo) targeting cassette.

The targeting vector linearized with NotI was electroporated into E14 (129 strain-derived) embryonic stem (ES) cells. Transfected cells were plated on neomycin-resistant, mitomycin C-treated mouse embryonic feeder cells, and positive selection was performed in the presence of Geneticin (G418; $125 \mu \mathrm{g} / \mathrm{ml})$. Homologous recombinant ES clones were confirmed by hybridizing the EcoRV- or SpeI-digested genomic DNA to a 356 bp (probe P2) or a 385 bp (probe P1) probe. Approximately 15 ES cells from each of the two targeted clones (A38 and A151) were injected into C57BL/6 blastocysts. Both ES clones produced several chimeras with $>50 \%$ agouti coat color. Two (one for each clone) male chimeras were bred to C57BL/ 6 females to allow for the detection of germ-line transmission. Heterozygous flox-neo female mice were bred to CAG-cre males, in which the cre gene is under control of the cytomegalovirus immediate early enhancer-chicken $\beta$-actin hybrid (CAG) promoter (Sakai and Miyazaki, 1997), to generate heterozygous knock-out mice. Recombination between two loxP sites was confirmed by hybridizing the HincII/MluI- or AccI-digested genomic DNA to a 383 bp (probe P3) or a $441 \mathrm{bp}$ (probe P4) probe. In this study, we used $\mathrm{Dscam}^{-/-}$mice and their littermates from 7 th to 16 th generations backcrossed to $\mathrm{C} 57 \mathrm{BL} / 6$, as well as mixed background of $50 \%$ C57BL/6-50\% BALB/c or $25 \%$ C57BL/6$75 \% \mathrm{BALB} / \mathrm{c}$ mice.

The experimental procedures and housing conditions for animals were approved by the RIKEN Animal Experiments Committee, and all of the animals were cared for and treated humanely in accordance with the Institutional Guidelines for Experiments using Animals.

Northern blot analysis. Brain tissues were dissected from postnatal day 0 (P0) mice. Total RNA was extracted using Trizol reagent (Invitrogen), and mRNA was isolated with a $\mu$ MACS mRNA Isolation kit (Miltenyi Biotec). Three micrograms of poly(A) RNA was loaded per lane on $1 \%$ agarose formaldehyde gel, and the size-separated RNA was transferred to a nylon membrane (Biodyne; Pall Bio Support). For hybridization, DNA probes were labeled with $\left[\alpha-{ }^{32} \mathrm{P}\right] \mathrm{dCTP}$ and hybridized overnight in ExpressHyb hybridization solution (Clontech), as recommended by the manufacturer. The blots were washed at a final concentration of $0.1 \times$ $\mathrm{SSC} / 0.1 \% \mathrm{SDS}$ at $55^{\circ} \mathrm{C}$ and exposed to $\mathrm{x}$-ray film at $-80^{\circ} \mathrm{C}$.

In vivo measurement of respiration in mouse. Whole-body plethysmography was performed on P0 mice within $24 \mathrm{~h}$ of birth (Kuwaki et al., 1996). $\mathrm{CO}_{2}$ response was examined by replacing the airflow through the plethysmograph with hypercapnic flows $\left(8 \% \mathrm{CO}_{2} / 92 \% \mathrm{O}_{2}\right)$ for $2 \mathrm{~min}$. Respiratory frequency, tidal volume, minute volume, and respiratory depth were calculated for $50-70$ respiratory cycles during quiet breathing. Naloxone was administered subcutaneously $(1,3.3$, or $10 \mu \mathrm{g} / \mathrm{g}$, respectively, in $20-30 \mu \mathrm{l}$ of saline) using a Hamilton syringe $\sim 6 \mathrm{~h}$ after the delivery from their mothers at embryonic day 19 (E19).

In vitro measurement of respiration and heart beat in mouse. For the respiration measurements, medulla and spinal cords for in vitro preparations were isolated from $\mathrm{P} 0$ or E19 mice under deep ether anesthesia as described previously (Shirasawa et al., 2000). Briefly, the brainstem was rostrally decerebrated between the VI cranial nerve roots and the caudal border of the trapezoid body so that most of the pons was removed. For the measurement of facial nerve activity, the caudal half of the pons was retained, attached to the facial nerve roots. Block was transferred to a $2 \mathrm{ml}$ recording chamber with continuous perfusion $(2 \mathrm{ml} / \mathrm{min})$ of modified Krebs' solution consisting of $124 \mathrm{~mm} \mathrm{NaCl}, 5.0 \mathrm{~mm} \mathrm{KCl}, 1.2 \mathrm{~mm} \mathrm{KH}_{2} \mathrm{PO}_{4}$, $2.4 \mathrm{mM} \mathrm{CaCl}_{2}, 1.3 \mathrm{mM} \mathrm{MgCl}_{2}, 26 \mathrm{~mm} \mathrm{NaHCO}_{3}$, and $30 \mathrm{~mm}$ glucose equilibrated with $95 \% \mathrm{O}_{2}$ and $5 \% \mathrm{CO}_{2}, \mathrm{pH} 7.4$, at $26-27^{\circ} \mathrm{C}$. Respiratorylike activity corresponding to the inspiration rhythm was monitored at the C4/C5 ventral root through a glass capillary suction electrode and a high-pass filter with a $0.3 \mathrm{~s}$ time constant. Nerve activity (bursts per minute) was calculated from the mean burst activity over a period of 3-5 min. Membrane potentials of inspiratory neurons in the rostral ventrolateral medulla (RVLM) (Arata et al., 1990) just caudal (within $200 \mu \mathrm{m}$ ) to the caudal end of the facial nucleus and overlapping the caudal part of the parafacial respiratory group (pFRG) (Onimaru and Homma, 2003) were recorded using conventional whole-cell patch-clamp methods (Onimaru and Homma, 1992). The membrane potentials were recorded with a current- and voltage-clamp amplifier (Axoclamp 2B; Molecular Devices) after compensation of the series resistance (25-50 M $\Omega$ ) and capacitance. Facial nerve activities were recorded by glass capillary suction electrodes through a high-pass filter with a $0.1 \mathrm{~s}$ time constant. For the heartbeat measurement, the previously described method (Paton, 1996) has been modified. Briefly, brain blocks attached with rib cages have been prepared, and heart rate was measured from exposed heart in the ribcage by using suction electrode.

Optical recordings of respiratory neuron activity. The medulla-spinal cord preparation was incubated in a modified Krebs' solution containing a fluorescent voltage-sensitive dye, Di-2-ANEPEQ $(33 \mu \mathrm{g} / \mathrm{ml}$; Invitrogen) for 30 min with $95 \% \mathrm{O}_{2}$ and $5 \% \mathrm{CO}_{2}$ equilibration at room temperature. After being stained, the preparation was placed with the ventral surface up in a $2 \mathrm{ml}$ perfusion chamber, which was mounted on a fluorescence microscope (THT-75 microscope setup; Brain Vision). Neuronal activity was detected using a voltage-sensitive dye with an optical recording apparatus (MiCAM02; Brain Vision) through a 510-550 nm excitation filter, a dichroic mirror, and a $590 \mathrm{~nm}$ absorption filter with a tungsten-halogen lamp $(150 \mathrm{~W})$ as the light source. The CCD-based camera consists of $192 \times 128$ pixels $(33.3 \times 37.5 \mu \mathrm{m} /$ pixel $)$. An optical image was obtained every $20 \mathrm{~ms}$. Acquisition time of inspiratory activity was $5.12 \mathrm{~s}$, consisting of $3.84 \mathrm{~s}$ before and $1.28 \mathrm{~s}$ after the initiation of the $\mathrm{C} 4$ inspiratory burst. Obtained images were averaged for 50 respiratory cycles. Each type of optical activity was determined as an averaged $\Delta F / F$ value containing 78 pixels. The fluorescence changes were expressed as a ratio (percentage, fractional changes) (i.e., the fluorescence intensity against that of the reference image). The differential image, processed with a software-spatial filter for $2 \times 2$ pixels, was represented by a pseudocolor display in which red corresponded to a fluorescence decrease, indicating membrane depolarization. BV Analyze (Brain Vision) was used for data acquisition and analysis.

Histological analyses. For measurement of the width of the cerebrum or medulla, we collected the brains from each of seven $D_{\text {scam }}{ }^{-1}$ mice and their wild-type littermates at E19 with immersion fixation. For analysis of the sectioned brain samples, we used E19 brain samples with perfusion fixation in each of six additional $D_{\text {scam }}{ }^{-1-}$ mice and their wild-type littermates. At the time the animals were killed on E19, pregnant dams were killed with overdose of ether, and embryos were removed from the uterus. After soaking embryos in cold PBS to stop the heart beating, embryos were transcardially perfused with $4 \%$ paraformaldehyde in 0.1 $\mathrm{M}$ phosphate buffer, $\mathrm{pH}$ 7.4. The heads were removed and immersed in the same fixative at $4^{\circ} \mathrm{C}$ for $4 \mathrm{~h}$ and then washed in several changes of PBS. After cryoprotection in $20 \%$ sucrose, the heads were soaked in OCT compound (Tissue-Tek; Sakura), placed in the desired orientation in molds made of aluminum foil, and then frozen on a metal block in a liquid nitrogen. The head was cut on a cryomicrotome (CM1900; Leica) in the transverse (frontal) plane. The sections were oriented as close as possible to the coronal plane on the basis of bilateral anatomical landmarks (hippocampus or several nuclei of the medulla). NIH ImageJ $1.33 \mathrm{u}$ software was used for the measurement of coronal sections of medulla. 
Brains were cut serially at $15 \mu \mathrm{m}$ in the coronal plane and collected in five adjacent series at $75 \mu \mathrm{m}$ intervals from the cerebral cortex to the area postrema. Every fifth section was stained with $0.1 \%$ toluidine blue for Nissl bodies. Immunohistochemistry was performed as follows: the slides were incubated with antibodies against nonphosphorylated neurofilament (1:2000; clone SMI311; Sternberger Monoclonals), calretinin (1:2000; Swant), or peripherin (1:200; Millipore Bioscience Research Reagents) for $1 \mathrm{~h}$ at room temperature and then washed for $2 \mathrm{~h}$ in several changes of PBS. Immunoreactivities were visualized using FITCconjugated (1:500; Vector Laboratories) or cyanine 3-conjugated (1:2000; Millipore Bioscience Research Reagents) secondary antibodies.

$m R N A$ in situ hybridization. Mouse brain at P0 was fixed by transcardial perfusion with ice-cold $4 \%$ paraformaldehyde in PBS, followed by overnight immersion at $4^{\circ} \mathrm{C}$ in the same fixative. Dissected brain was embedded in $10 \%$ gelatin (Sigma). The embedded and trimmed block was refixed with $4 \%$ paraformaldehyde in PBS and cryoprotected by immersion in $20 \%$ sucrose in PBS. Sections were cut frozen on a cryostat at $60 \mu \mathrm{m}$, collected in plastic vials containing PBS, dehydrated through a graded methanol series in PBS-Tw (PBS with $0.1 \%$ Tween 20), and then stored in $100 \%$ methanol at $-20^{\circ} \mathrm{C}$ until required. The $1.9 \mathrm{~kb}$ fragments of mouse Dscam cDNA corresponding to nucleotides 3753-5694 (GenBank accession number NM_031174) subcloned into the pBluescript II $\mathrm{SK}(-)$ vector was used as template for synthesizing the antisense cRNA probes. Antisense cRNA probes were synthesized using a DIG RNA labeling kit with T3 RNA polymerase (Roche Diagnostics). Free-floating sections were bleached with $6 \% \mathrm{H}_{2} \mathrm{O}_{2}$ in methanol for $1 \mathrm{~h}$ at room temperature. After three 5 min washes in PBS-Tw, sections were digested for $5 \mathrm{~min}$ with $10 \mu \mathrm{g} / \mathrm{ml}$ proteinase $\mathrm{K}$ in PBS-Tw, followed by 20 min postfixation at room temperature with $4 \%$ paraformaldehyde in PBS and three 5 min washes in PBS-Tw. Sections were prehybridized in hybridization mix without cRNA probe for $1 \mathrm{~h}$ at $68^{\circ} \mathrm{C}$ and then hybridized overnight $\left(>12 \mathrm{~h}\right.$ ) at $68^{\circ} \mathrm{C}$. The hybridization mix was composed of $50 \%$ formamide, $5 \times$ SSC, $0.1 \%$ Tween $20,100 \mu \mathrm{g} / \mathrm{ml}$ heparin (Sigma), and $200 \mu \mathrm{g} / \mathrm{ml}$ yeast tRNA (Invitrogen). Probe concentration was $\sim 100 \mathrm{ng} /$ $\mathrm{ml}$. After hybridization, sections were washed twice for $20 \mathrm{~min}$ at $63^{\circ} \mathrm{C}$ in $50 \%$ formamide, $5 \times$ SSC, $0.1 \%$ Tween 20 , followed by three $20 \mathrm{~min}$ washes at $63^{\circ} \mathrm{C}$ in $50 \%$ formamide, $1 \times$ SSC, $0.1 \%$ Tween 20 . Labeled cRNA probe hybridized to the section was detected using sheep antidigoxigenin Fab fragments conjugated to alkaline phosphatase (1:5000; Roche Diagnostics) and nitroblue-tetrazolium-chloride/5-bromo-4chloro-3-indolyl-phosphate as a chromogenic substrate.

Neurite growth assay. Two chimeric proteins that consist of the whole extracellular domains of CAMs (human DSCAM and human L1CAM, respectively) and the $\mathrm{Fc}$ region of human IgG were used in the present study. The pIg vector containing an insert of human L1CAM-Fc was described previously (Fransen et al., 1998). Human DSCAM-Fc was constructed using human DSCAM cDNA, cDSC1 (GenBank accession number AF217525), and the pIg-tail expression system (Novagen). A cDNA encoding the extracellular domain of human DSCAM (nucleotides 4825263) was obtained by PCR from the pBluescript II SK(-), which contains a cDNA encoding the full-length human DSCAM in its EcoRI ( $\left.5^{\prime}\right)$ and $\mathrm{XhoI}\left(3^{\prime}\right)$ sites. Primers used for the PCR amplification were as follows: a sense primer, $5^{\prime}$-CGCTCTAGATGGATACTGGCTCTCTCCTT-3'; and an antisense primer, 5'-TGAGCGGCCGCCATCTTGAGCCCCTCGTTG- $3^{\prime}$. The sense primer contains a cDNA fragment (nucleotides 482-501) encoding the $\mathrm{N}$-terminal end of the DSCAM extracellular domain, which is immediately preceded by an $\mathrm{XbaI}$ restriction site. The antisense primer contains a cDNA fragment (nucleotides 5245-5263) encoding the C-terminal end of the DSCAM extracellular domain, which is immediately followed by an NotI restriction site. The PCR product with sense and antisense primers was digested with $\mathrm{XbaI}$ (also located at nucleotide 990 of human DSCAM) and NotI and ligated into an $\mathrm{XbaI}-$ NotI-digested pIg vector. The PCR-amplified region and the insert-vector junctions were confirmed by sequencing.

Dorsal root ganglia (DRGs) dissected from P0 or E19 mice were treated with $0.25 \%$ trypsin-EGTA (Invitrogen) at $37^{\circ} \mathrm{C}$ for $30 \mathrm{~min}$ and dissociated by trituration using the $\mathrm{p} 20$ micropipetman. The dissociated cells were resuspended in RPMI-1640 medium (Invitrogen) supplemented with $10 \%$ fetal bovine serum (Invitrogen), 1\% penicillin-strep-
tomycin-Fungizone (PSF) (Invitrogen), and $20 \mathrm{ng} / \mathrm{ml}$ nerve growth factor (Promega) and plated on a glass-base dish coated with DSCAM-Fc or L1CAM-Fc. CAM-Fc-coated dishes were prepared by sequential coating with $0.1 \mathrm{mg} / \mathrm{ml}$ poly-D-lysine (70-150 kDa; Sigma-Aldrich), $40 \mu \mathrm{g} / \mathrm{ml}$ anti-Fc antibody (Sigma-Aldrich) and CAM-Fc. Production of CAM-Fc was performed as described previously (Kamiguchi and Yoshihara, 2001). The cells were cultivated in a humidified incubator at $5 \% \mathrm{CO}_{2}$ and $37^{\circ} \mathrm{C}$ for $7 \mathrm{~h}$ before fixation with $4 \%$ formaldehyde.

Images of DRG neurons taken with a cooled digital CCD camera (CoolSNAP HQ; Photometrics) were analyzed using MetaMorph version 4.6 (Universal Imaging). Neurite length was measured as the distance between the tip of the longest neurite and the periphery of the cell soma in which the neurite emerges.

Statistical analysis. Statistical analyses of these data were performed by Student's $t$ test, and the Tukey-Kramer test was used for multiple comparisons. The data are expressed as mean \pm SEM, and $p<0.05$ was considered as statistically significant.

\section{Results}

\section{Neonatal death of Dscam-deficient mouse}

To explore the physiological functions of DSCAM protein, we generated Dscam-deficient mice (Fig. 1). The first exon of Dscam gene contains the flanking 5' untranslated region, codon for the first methionine, and codon for the $\mathrm{N}$-terminal signal peptide sequence of DSCAM protein. We constructed a targeting vector to disrupt the first exon using the cre-loxP system (Fig. 1 $A, B$ ). To generate mice with heterozygous deletion of the first exon, mice with the flox-neo allele derived from two independent ES clones were bred with CAG-cre mice. The heterozygous knock-out $\left(\right.$ Dscam $\left.^{+/-}\right)$mice were fertile and developed almost normally. Matings of heterozygotes $\left(\right.$ Dscam $\left.^{+/-}\right)$yielded homozygotes $\left(D_{s c a m}^{-1-}\right)$. Northern blot analysis revealed an absence of Dscam transcripts in the brains of $D s c a m^{-/-}$pups (Fig. 1C). RNA in situ hybridization of Dscam ${ }^{-/-}$pups also showed no Dscam transcripts, whereas the broad expression of Dscam in the brain, including hippocampus, olfactory bulb, cerebral cortex, and medulla, was detected in the wild-type littermates at P0 (data not shown). Dscam ${ }^{+/-}$mothers delivered their pups later than the expected day in more than half of the pregnancies. Those delayed deliveries often let the mothers kill their pups. We therefore killed mothers if their pregnancies exceeded $19 \mathrm{~d}$ and delivered pups by cesarean section (E19). No recognizable differences were observed between naturally born mice and the surgically delivered mice $\left(\right.$ Dscam $^{+/-}$, Dscam $^{-/-}$, and wild-type littermates) in the following experiments. Populations of pups for each genotype were in accordance with the expected Mendelian distribution: 162 wild-type littermates (26.1\%), $321 \mathrm{Dscam}^{+/-}$(51.8\%), and $137 \mathrm{Dscam}^{-1-}(22.1 \%)$ of 620 mice in total. This indicates that deficiency of DSCAM (Dscam ${ }^{-1-}$ ) does not lead to embryonic lethality. However, a majority of the null mutant mice $\left(\right.$ Dscam $\left.^{-1-}\right)$ died within $24 \mathrm{~h}$ after birth and rarely survived beyond $48 \mathrm{~h}$. The $D s \mathrm{sam}^{-/-}$pups appeared to be normal at birth, and their body weights were not statistically different compared with wild-type or Dscam ${ }^{+/-}$littermates (data not shown). Most Dscam $^{-1-}$ mice did not have milk in their stomachs but some did, indicating that $\mathrm{Dscam}^{-1-}$ pups can nurse. The wild-type littermates grew normally, indicating that the $\operatorname{Dscam}^{+/-}$mothers can foster or raise their pups normally. We did not observe any difference between the lifespans of wild-type and $D_{\text {scam }}^{+/-}$pups. Furthermore, we have not observed any significant abnormalities in Dscam $^{+/-}$mice except for the moderately abnormal respiration that is described below. In the following experiments, we used the Dscam ${ }^{-/-}$mice at E19 (surgically delivered pups) as well as at $\mathrm{P} 0$ (naturally born pups). 
A

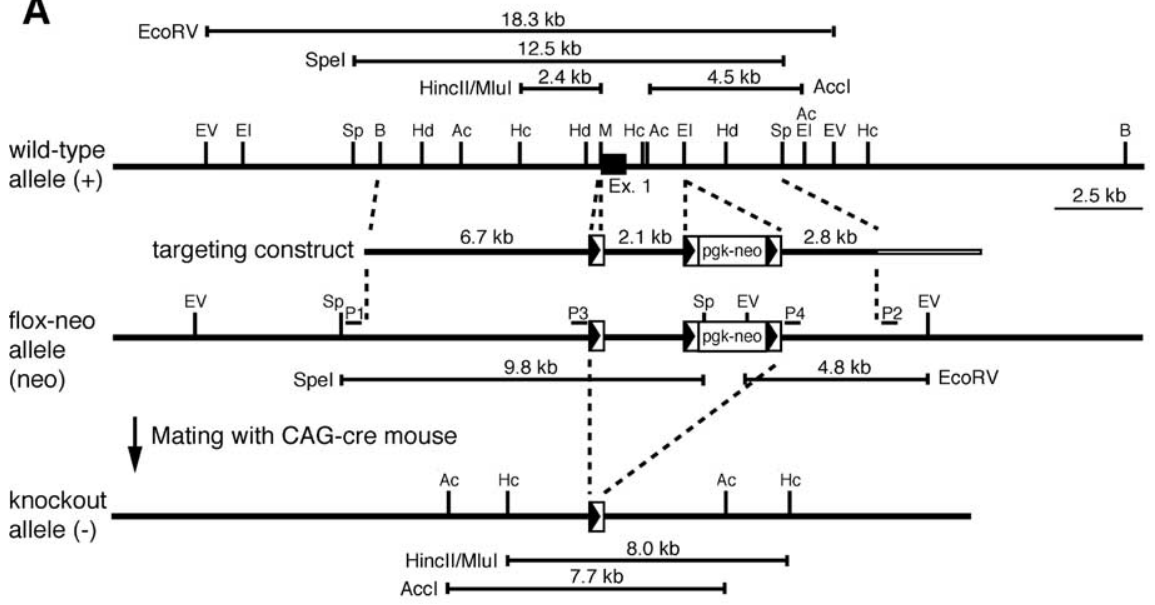

B

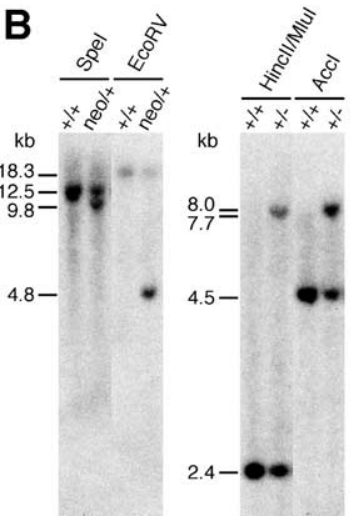

C
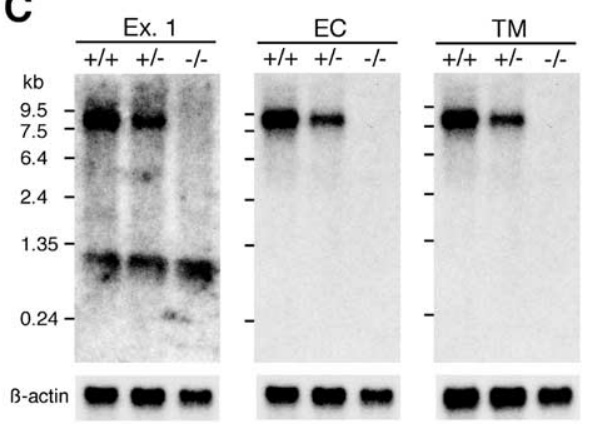

Figure 1. Targeted disruption of Dscam in mice. $A$, Strategy for generating the targeted Dscam allele. Schematic representation of wild-type Dscam locus including exon 1 (Ex. 1, solid box), targeting construct using the cre-loxP system, initial homologously recombined flox-neo allele, and targeted Dscam knock-out allele. To obtain the exon 1-deleted knock-out allele, heterozygous mice with a flox-neo allele were mated with the CAG - cre mice. Four probes (P1, P2, P3, and P4) used for Southern blot analyses are indicated. Ac, AcCl; B, BamHI; El, EcoRl; EV, EcoRV; Hc, Hincll; Hd, Hindlll; M, Mlul; Sp, Spel. B, Southern blot analysis of Spel- or EcoRV-digested genomic DNA from wild-type $(+/+$ ) or flox-neo (neo/+) mice revealed 9.8 or $4.8 \mathrm{~kb}$ fragments in homologous recombinant flox-neo mice; Hincll/Mlul- or Accl-digested genomic DNA from wild-type $(+/+)$ or heterozygous $(+/-)$ mice revealed 8.0 or $7.7 \mathrm{~kb}$ fragments in exon 1-deleted heterozygous mice. $\boldsymbol{C}$, Northern blot analysis of RNA extracted from the brains of wild-type, Dscam ${ }^{+/-}$, and Dscam ${ }^{-/-}$mice at PO using four parts of Dscam CDNA [Ex. 1, exon 1 (nucleotides 1-496); EC, extracellular (nucleotides 2299-2626); TM, transmembrane (nucleotides 5210-5410); (P, cytoplasmic (nucleotides $5385-5637)$ ] as probes. All four probes detected the $8.7 \mathrm{~kb}$ Dscam transcript in the wild-type and Dscam ${ }^{+/-}$mice. Note that the targeted transcripts disappeared completely in Dscam ${ }^{-1-}$ mice. The exon 1 probe detected a nonspecific band of $1.0 \mathrm{~kb}$ in addition to the $8.7 \mathrm{~kb}$ Dscam transcript. $\beta$-Actin probe was used as a control.

\begin{abstract}
Dscam $^{-/-}$mice show irregular central respiratory activities Dscam ${ }^{-/-}$pups occasionally showed jerky or dyspnea-like movements (supplemental Movie 1, available at www.jneurosci.org as supplemental material). Whole-body plethysmography showed irregular respiratory rhythm and frequent apneic episodes in Dscam ${ }^{-1-}$ pups, whereas wild-type littermates showed regular and constant breathing (Fig. 2A-D). The heterozygotes $\left(\right.$ Dscam $\left.^{+/-}\right)$showed similar but less serious respiratory abnormalities. To investigate whether the abnormal respiratory rhythm in Dscam ${ }^{-1-}$ pups was attributable to defects of the respiratory center in the brain, we recorded $\mathrm{C} 4$ ventral root activity in a medulla-spinal cord preparation as a measure of respiratory output (Arata et al., 1990). The C4/C5 activity is known to be synchronous with phrenic nerve discharge and contraction of the diaphragm and inspiratory intercostal muscles. C4 ventral root recordings in Dscam $^{-/}$mice showed irregular activity (frequency per minute, $4.1 \pm 0.6 ; n=11)$ compared with those seen in wild-type mice $(6.8 \pm 0.4 ; n=7)(p<0.05)$ (Fig. $3 A, B)$.
\end{abstract}

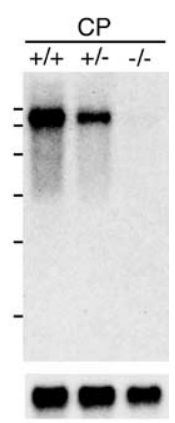

Continuous low-amplitude C4 activities representing apnea were often observed in Dscam ${ }^{-1-}$ mice that well correlated with the lethality (Fig. $3 A$, arrows, $C$ ). The $\mathrm{C} 4$ activity of $D$ scam ${ }^{+/-}$mice (frequency per minute, $7.3 \pm 0.8 ; n=8$ ) was also irregular but less severe than that of $D$ scam ${ }^{-1-}$ mice (Fig. $3 A, B$ ). Because a medulla-spinal cord preparation lacks centripetal inputs from the peripheral stretch receptors or chemoreceptors, the frequency of $\mathrm{C} 4$ inspiratory activity in vitro is less than that of respiratory rhythm in vivo. We further checked the heart beats of $D$ scam ${ }^{-1-}$ pups (frequency per minute, $19.6 \pm 0.9 ; n=4$ ), which were almost identical to that of wild-type pups $(21.1 \pm 0.5 ; n=4 ; p=$ $0.19)$. These results suggest that the abnormal respiration patterns in $D s c a m^{-1-}$ and Dscam ${ }^{+/-}$mice were caused by defects of the central respiratory rhythm generator located in ventral medulla.

\section{Abnormal ventilatory response to hypercapnia in Dscam $^{-/-}$mouse} We further examined the ventilatory response to hypercapnia in $D_{\text {scam }}{ }^{-1-}$ and Dscam $^{+/-}$mice and their wild-type littermates at P0, using whole-body plethysmography (Fig. 4). The averages of respiratory frequencies were calculated only in the durations of continuous respiration without apnea (supplemental Tables 1, 2, available at www.jneurosci.org as supplemental material). Respiratory frequency under hypercapnia was increased in wildtype and Dscam ${ }^{+/-}$mice but was markedly decreased in $\mathrm{Dscam}^{-1-}$ mice (Fig. $4 A, B)$. Specifically, excess expiration in Dscam $^{+/-}$that is shown by the traces below the baseline became dominant. This may further suggest that $D$ scam ${ }^{+/-}$is responding to hypercapnia in a specific way. Continuous exposure to hypercapnia let the $D \mathrm{scam}^{-1-}$ mice die with a gradual decrease in respiratory amplitude. These results indicate that the $D_{\text {scam }}{ }^{-1-}$ (and $D$ scam ${ }^{+/-}$) mice show abnormal ventilatory responses to hypercapnia. The maintained respiratory frequencies in Dscam mutants in the air condition are possibly explained by compensation with feedback of peripheral chemoreceptors and pulmonary stretch receptors active in vivo and eliminated in the in vitro $\mathrm{C} 4$ measurement. However, it should be noted that the Dscam mutants show frequent apnea (Fig. 2).

\section{Pre-I neuron activity is deficient in $\mathrm{Dscam}^{-/-}$mouse}

Dual respiratory rhythm generators have been suggested to exist in the RVLM, one in the retrotrapezoid nucleus/pFRG containing pre-inspiratory (Pre-I) neurons (Feldman et al., 2003; Onimaru and Homma, 2003) and the other in the pre-Bötzinger complex (preBötC) containing inspiratory (Insp) neurons (Smith et al., 1991; Rekling and Feldman, 1998) (Fig. 5A,B). Activities of both rhythm generators have been reported to be mutually related (Onimaru et al., 2006). To visualize the spatio- 
temporal pattern of respiratory neuron activity in the ventral medulla, we used an optical recording system that uses a brainstem-spinal cord preparation stained with a voltage-sensitive dye (Onimaru and Homma, 2003; Onimaru et al., 2004). To reduce the input of inhibitory neuronal activity from the pons, the rostral limit of the medulla-spinal cord preparation was set at the middle of facial (VII) nucleus (Fig. $5 A$ ). Accumulated neuronal activities of 50 respiratory cycles, each of which was triggered by its $\mathrm{C} 4$ inspiratory activity on one side, were shown in images (Fig. $5 \mathrm{C}-\mathrm{H}$ ) and trajectories (Fig. $5 I-K$ ). In wild-type mice, we confirmed the patterns of two rhythm generator loci, Pre-I and Insp neurons, on each side (four loci in total) (Fig. $5 C, F)$. As reported previously (Onimaru et al., 2004), the averaged membrane potential trajectory of the Pre-I neuron activity appeared several hundred milliseconds before the onset of the Insp nerve activity in a limited region of the pFRG (Onimaru and Homma, 2003) (Fig. 5C,I) (supplemental Movie 2, available at www.jneurosci.org as supplemental material). Subsequently, the Insp neuron activity appeared to propagate medially and caudally in the ventral medulla (preBötC) (Fig. 5F,I). In contrast, the averaged membrane potential trajectory of the Pre-I neuron activity in the pFRG during the pre-inspiratory phase was reduced in Dscam $^{+/-}$mice (Fig. $5 D, J$ ) and disappeared in $\mathrm{Dscam}^{-1-}$ mice (Fig. $5 E, K, L$ ) (supplemental Movies 3, 4, available at www.jneurosci.org as supplemental material), although the Insp neuronal activities in preBötC during the inspiratory phase were essentially retained in both the genotypes (Fig. 5G, H,J,K). We further performed whole-cell patch-clamp analyses on pFRG on mouse brainstem-spinal cord preparations. As reported previously (Onimaru et al., 2006), our results in wildtype mice clearly showed both Pre-I neurons, which synchronously activate before the inspiratory phase, and Insp neurons, which synchronously activate at the C4 phase. In $D_{s c a m}{ }^{-1}$ mice, however, the synchronous Insp neuronal activities were found to be weak, and the neurons showing synchronous Pre-I neuronal activities were not observed in pFRG. Instead, in the pFRG, we detected some neurons with spontaneous although irregular activities (data not shown). We assume that these neurons could be originally Pre-I neurons, and the optical imaging data indicates that these neurons did not synchronize to each other and to the $\mathrm{C} 4$ activities.

Pre-I neuron activity has been reported to correlate with facial nerve activity in the
A

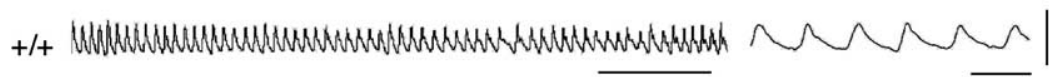

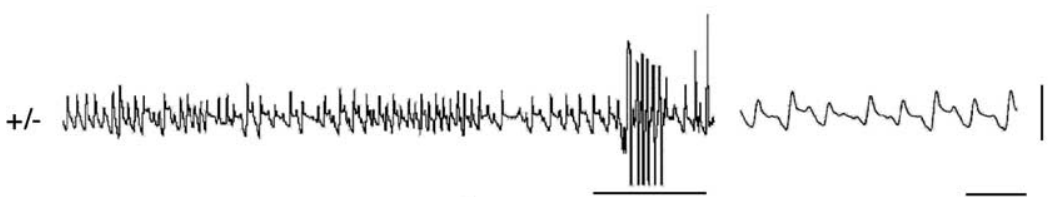
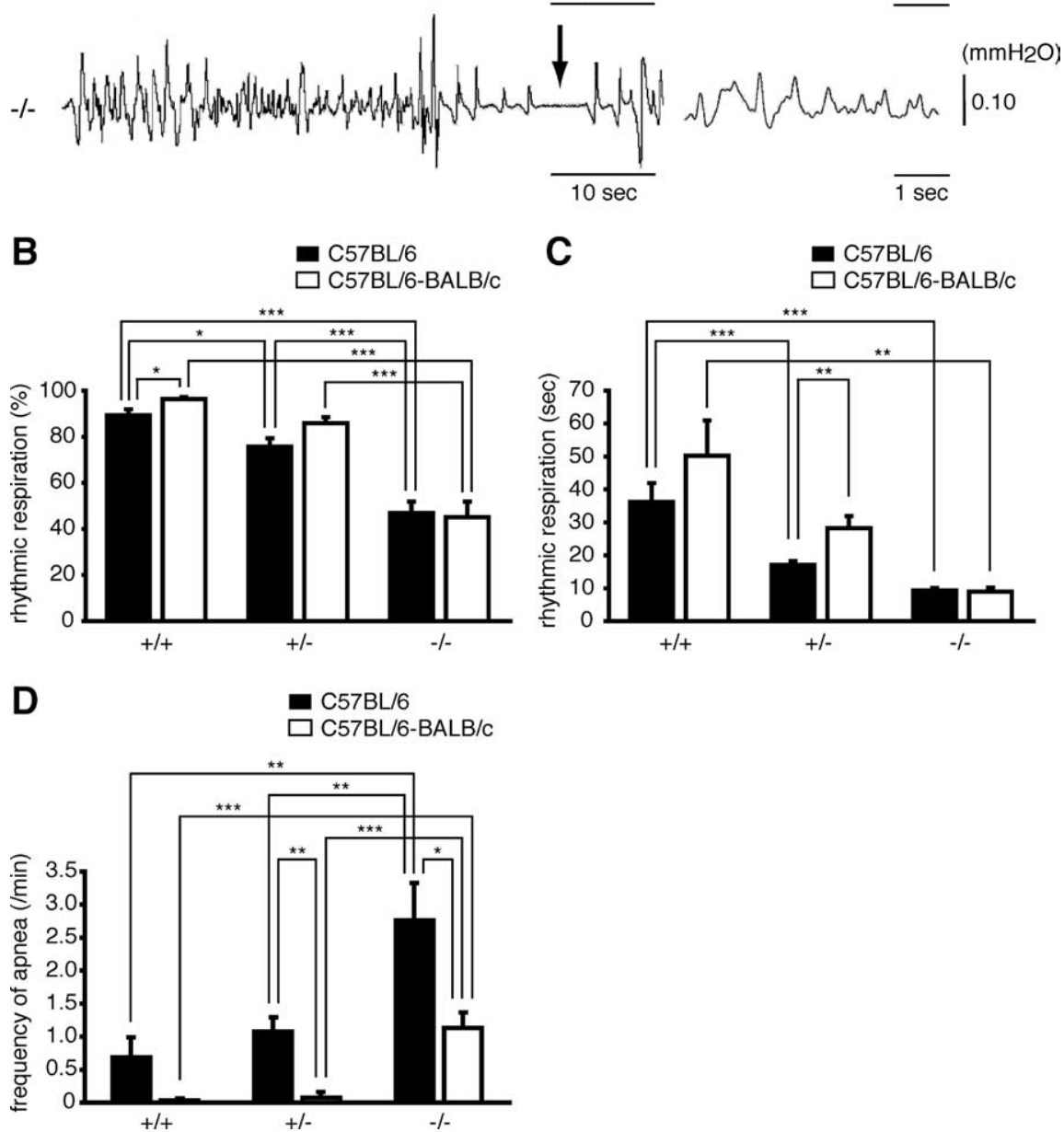

Figure 2. Dosage-dependent in vivo respiratory abnormalities in Dscam-deficient mice. Spontaneous respiration activities of newborn mice were measured by plethysmography. $A$, Recordings correspond to spontaneous respiration in wild-type, Dscam $^{+/-}$, and Dscam ${ }^{-/-}$littermates, respectively (C57BL/6 background; for C57BL/6-BALB/c mixed background, see Fig. $8 \mathrm{~A}$ ). Horizontal bar under each respiration activity represents a period of $10 \mathrm{~s}$ (left side) or $1 \mathrm{~s}$ (right side), respectively. Vertical bar at the right side of respiration activity represents a volume of $0.10 \mathrm{mmH}_{2} 0$. Dscam $^{-/}$mice show irregular patterns, and $\mathrm{Dscam}^{+/-}$also show similar but less severe respiratory abnormalities. In addition, continuous lack of respiration activity representing apnea was often observed in Dscam ${ }^{-1-}$ mice (arrow). B, C, Ratio of rhythmic respirations was quantified as percentage (rhythmic vs irrhythmic) or averaged time periods of continuous rhythmic respiration during quiet breathing without moving. The ratio (percentage) of rhythmic respiration $(\boldsymbol{B})$ or averaged continuous period of rhythmic respiration $(\boldsymbol{C})$ on $557 \mathrm{BL} / 6$ (filled bar) or C57BL/6-BALB/c mixed background (open bar) were calculated in wild-type $(n=10$ or $n=8)$, Dscam $^{+/-}(n=14$ or $n=9)$, and Dscam ${ }^{-1-}$ genotypes $(n=9$ or $n=7)$, respectively. $\boldsymbol{B}$, The ratio of rhythmic respiration in Dscam ${ }^{-/}$- mice was significantly lower than that in wild-type or Dscam ${ }^{+/-}$mice on both C57BL/6 and C57BL/6 -BALB/c mixed backgrounds. In Dscam wild-type genotype, the ratio of rhythmic respiration on $\mathrm{C} 57 \mathrm{BL} / 6$ - BALB/C mixed background was close to but still statistically higher than that on C57BL/6 background. C, The averaged time period of continuous rhythmic respiration in Dscam ${ }^{-1-}$ mice was significantly shorter than that in wild-type mice on both $57 \mathrm{BL} / 6$ and $\mathrm{C} 57 \mathrm{BL} / 6$-BALB/c mixed backgrounds. The averaged period of rhythmic respiration in Dscam ${ }^{+-}$mice was also significantly shorter than that in wild-type mice on $\mathrm{C57BL} / 6$ background. Note that the period of rhythmic respiration in $\mathrm{Dscam}^{+/-}$mice on $\mathrm{C} 57 \mathrm{BL} / 6-\mathrm{BALB} / \mathrm{c}$ mixed background was significantly longer than that in Dscam $^{+/-}$mice on C57BL/6 background. The period of rhythmic respiration in Dscam wild type on C57BL/6 -BALB/c mixed background was longer than that in $\mathrm{Dscam}^{+/-}$mice on $\mathrm{C} 57 \mathrm{BL} / 6$ background but statistically not significant. $\boldsymbol{D}$, Frequency of apnea on C57BL/6 (filled bar) or C57BL/6-BALB/c mixed background (open bar) was quantified as the number of apnea that continued without respiration for $>1$ sin wild-type $(n=10$ or $n=9)$, Dscam $^{+/-}(n=14$ or $n=9)$, and Dscam ${ }^{-1-}$ genotypes ( $n=9$ or $n=7$ ), respectively. The frequency of apnea in $D_{\text {scam }}{ }^{-1-}$ mice was significantly higher than (Figure legend continues.) 
A
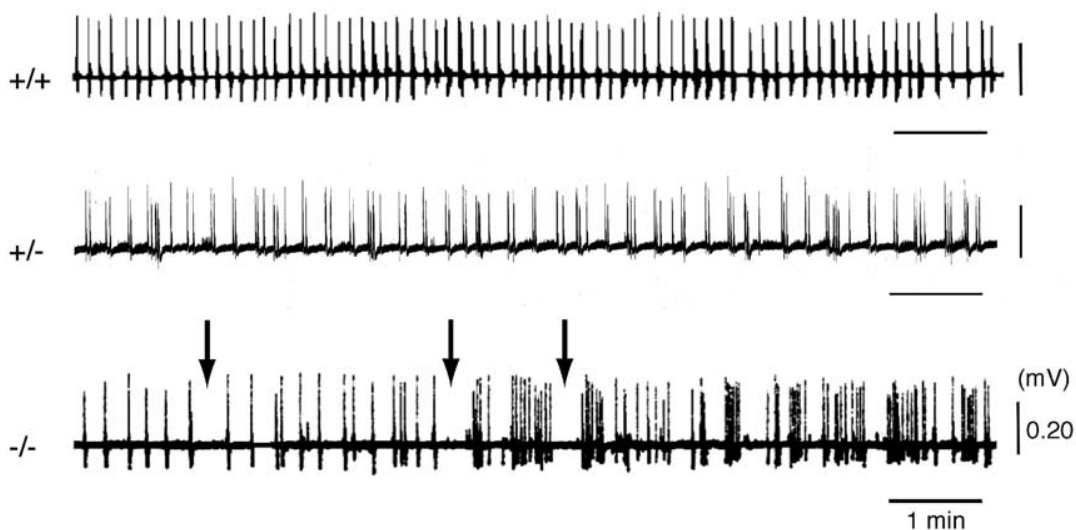

B

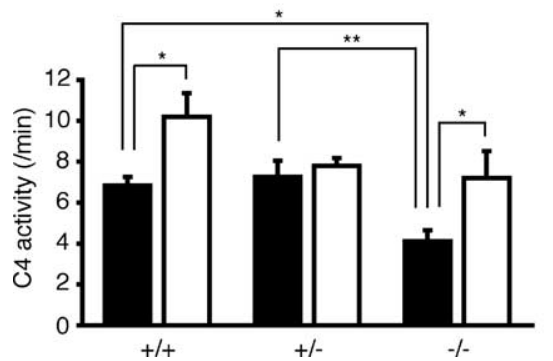

C

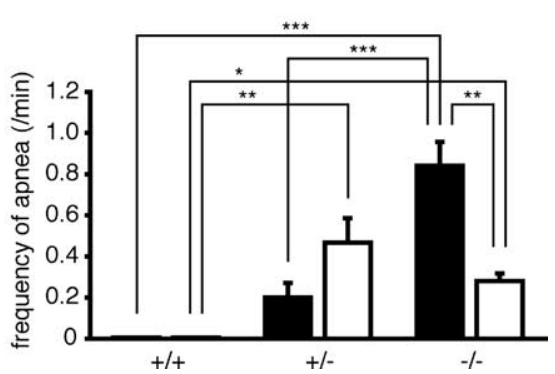

Figure 3. Dosage-dependent abnormalities of in vitro inspiratory activity in Dscam-deficient mice. (4 ventral root inspiratory activities in medulla-spinal cord preparations of newborn mice were measured. $\boldsymbol{A}$, Recordings correspond to $\mathrm{C} 4$ inspiratory activity in wild-type, Dscam ${ }^{+/-}$, and Dscam ${ }^{-/-}$littermates, respectively (C57BL/6 background; for C57BL/6-BALB/c mixed background, see Fig. 8 B). Horizontal bar under each (4 activity represents a period of $1 \mathrm{~min}$. Vertical bar at the right side of $C 4$ activity represents an amplitude of $0.20 \mathrm{mV}$. The activities for Dscam ${ }^{-1-}$ show irregular patterns. Lack of large C4 inspiratory activities representing apnea was often observed in Dscam ${ }^{-1-}$ mice (arrows). The activities of Dscam ${ }^{+/-}$mice were also irregular but less severe than that of $D$ scam ${ }^{-/-}$mice. $B$, Nerve activity (bursts per minute) on $C 57 \mathrm{BL} / 6$ (filled bar) or $C 57 \mathrm{BL} / 6-$ $\mathrm{BALB} / \mathrm{c}$ mixed background (open bar) was calculated in wild-type $(n=7$ or $n=5), \operatorname{Dscam}^{+/-}(n=8$ or $n=5)$, and Dscam ${ }^{-/-}$ genotypes ( $n=11$ or $n=5$ ), respectively. The frequency of (4 inspiratory activity in Dscam ${ }^{-1-}$ mice was significantly less than that in wild-type or Dscam ${ }^{+/-}$mice on C57BL/6 background. The (4 activity in Dscam ${ }^{+/-}$and Dscam ${ }^{-1-}$ mice on C57BL/6$B A L B / c$ mixed background was less frequent than that in wild-type mice but statistically not significant. C, Frequency of apnea, which was defined as lack of large (4 inspiratory activities for $>15 \mathrm{~s}$, on C57BL/6 (filled bar) or C57BL/6-BALB/c mixed background (open bar), was quantified in wild-type $(n=5$ or $n=3)$, Dscam $^{+/-}(n=5$ or $n=3)$, and Dscam ${ }^{-/-}$genotypes $(n=$ 5 or $n=5$ ), respectively. The frequency of apnea in $D_{s c a m}{ }^{-1-}$ mice was significantly higher than that in wild type on both $\mathrm{C} 57 \mathrm{BL} / 6$ and $\mathrm{C57BL} / 6-\mathrm{BALB} / \mathrm{c}$ mixed backgrounds. That of Dscam ${ }^{-/-}$was also significantly higher than that in Dscam ${ }^{+/-}$on C57BL/6 background. Although the frequency of apnea in Dscam ${ }^{-/-}$was lower than that in Dscam ${ }^{+/-}$on C57BL/6-BALB/C mixed background, the difference was not statistically significant. Note that the frequency of apnea in Dscam ${ }^{-1-}$ on $C 57 \mathrm{BL} / 6$ background was significantly higher than that on $\mathrm{C57BL} / 6-\mathrm{BALB} / \mathrm{c}$ mixed background. No apnea was found in wild-type mice on both C57BL/6 and C57BL/6 -BALB/c mixed backgrounds. Statistics on Dscam genotypes with three entities were calculated by Tukey-Kramer test. Those on strains with two entities (C57BL/6 and C57BL/6-BALB/c mixed backgrounds) were calculated by Student's $t$ test. ${ }^{*} p<0.05,{ }^{* *} p<0.01$, and ${ }^{* * *} p<0.001$. Error bars represent SEM.

newborn rat brainstem-spinal cord preparation (Onimaru et al., 2006). We therefore investigated the output of the facial nerve activity together with C4 inspiratory activity in $\mathrm{Dscam}^{-1-}$ and wild-type mice using the preparation with more rostral anterior

\section{$\leftarrow$}

(Figure legend continued.) that in wild-type or Dscam ${ }^{+/-}$mice on both $557 \mathrm{BL} / 6$ and $\mathrm{C57BL} /$ $6-B A L B / c$ mixed backgrounds. Note that the frequency of apnea in $D$ scam ${ }^{-1-}$ mice on C57BL/6 background was significantly higher than that in Sscam $^{-1-}$ mice on C57BL/6$\mathrm{BALB} / \mathrm{c}$ mixed background. Statistics on Dscam genotypes with three entities were calculated by Tukey-Kramer test. Those on strains with two entities (C57BL/6 and C57BL/6-BALB/C mixed backgrounds) were calculated by Student's $t$ test. ${ }^{*} p<0.05$, ${ }^{* *} p<0.01$, and ${ }^{* * *} p<$ 0.001. Error bars represent SEM. limit (Fig. $5 A$, open arrow) to keep the facial nucleus intact. In wild-type mice, the activities of the facial nerve were regular, constantly phasic, and roughly synchronized with their $\mathrm{C} 4$ inspiratory activities $(n=6)$, whereas that of $D s c a m^{-1-}$ mice became tonic and lost their synchroneity to the $\mathrm{C} 4$ activities $(n=5)$ (Fig. $5 M)$. In this study, we did not examine the Pre-I neuronal activity at the measurement of facial nerve activity.

Naloxone, an antagonist for opioid receptors, has been reported to relieve the lethality in $\mathrm{Krox} 20^{-/-}$, another mouse model of central respiratory defect, and lacks rhombomere 3 and 5 that develop to the region containing pFRG (Jacquin et al., 1996). We subcutaneously administrated multiple doses of naloxone to Dscam ${ }^{-1-}$ mice ( $n=3$ for $1 \mu \mathrm{g} / \mathrm{g}$ body weight; $n=2$ for $3.3 \mu \mathrm{g} / \mathrm{g} ; n=2$ for $10 \mu \mathrm{g} / \mathrm{g})$. However, none of the Dscam $^{-1-}$ mice could survive beyond $24 \mathrm{~h}$ after birth. We found no difference in phenotypes before versus after administration of naloxone in Dscam $^{+/-}$ and wild-type mice.

\section{Homophilic binding of Dscam accelerates neurite extension} DSCAM protein mediates homophilic intercellular adhesion (Agarwala et al., 2000). Here we investigated the effect of homophilic binding of DSCAM on neurite extension by using DRG neuron culture, which have been well used to assess the neurite extension activities of Ig superfamily members (Nakata and Kamiguchi, 2007) and is known to well express DSCAM (Yamakawa et al., 1998; Barlow et al., 2002) and L1CAM (Kamiguchi and Yoshihara, 2001). Neurite outgrowth of cultured neonatal (P0 or E19) DRG neurons from $D_{\text {scam }}{ }^{-/-}$mice and their wildtype control littermates was analyzed on DSCAM- or L1CAM-coated dishes. The results of experiments performed on E19 are summarized in Figure 6. A similar result was obtained in litters on P0 (data not shown). The neurite length of DRG neurons on a DSCAM-coated dish was significantly longer than that on negative control dishes (Fig. 6A). We also observed that the neurite length of $\mathrm{Dscam}^{-1-}$ DRG neurons on a DSCAM-coated dish was significantly shorter compared with that of wild-type DRG neurons (Fig. 6A), whereas the percentage of DRG neurons bearing neurites did not differ between $\mathrm{Dscam}^{-1-}$ and wild-type DRG neurons (Fig. 6B). These results indicate that the homophilic binding of Dscam protein accelerates neurite extension, and we expect that its deficiency may lead to abnormal neural network formations.

\section{Enlarged medulla of Dscam ${ }^{-/-}$mouse}

Gross anatomical examination of $\mathrm{Dscam}^{-1-}$ mice at E19 revealed a significant enlargement of the mediolateral width of the me- 
dulla compared with littermate controls, whereas that of cortex mostly remained unchanged (Fig. 7 $A, B$ ). Light microscopic observation of Nissl-stained transverse sections confirmed the increased size of Dscam $^{-/-}$medulla at E19 (Fig. $7 C-G$ ). The cell density remained the same, therefore suggesting that the total cell number had increased. The enlargement was prominent in $\mathrm{Dscam}^{-/-}$ mice, especially at the area dorsolateral to the facial nucleus (Fig. $7 H-K$ ) (supplemental Fig. $1 A-H$, available at www. jneurosci.org as supplemental material). Immunohistochemical analysis with SMI311 (monoclonal antibody to panneuronal nonphosphorylated neurofilament), a marker for neuronal soma and dendrites, revealed increases of immunosignals in this area dorsolateral to the facial nucleus, indicating that the number of neurons had increased (Fig. $7 L-O)$ (supplemental Fig. $1 A^{\prime}-H^{\prime}$, available at www.jneurosci.org as supplemental material). These abnormal neurons may possibly lead to the deficient output of the facial nerve activity in Dscam $^{-1-}$ mice (Fig. 5M). Even with these abnormalities of medulla, the general structure of the Dscam ${ }^{-1-}$ brainstem as well as most of other immunosignals, including calretinin (interneuronal marker) and peripherin (type III neuronal intermediate filament), look similar to that of wild-type mice (supplemental Fig. $1 A^{\prime \prime}-H^{\prime \prime}, A^{\prime \prime \prime}-$ $H^{\prime \prime \prime}$, available at www.jneurosci.org as supplemental material). So far, we have not observed gross anatomical changes in other parts of the brain in $D$ scam ${ }^{-1-}$ mice, including cerebral cortex, hippocampus, thalamus, or amygdala (data not shown).

\section{Distinct phenotype of Dscam-deficient mouse in a different genetic background}

Although the $\mathrm{Dscam}^{-1-}$ mice in the C57BL/6 background died neonatally, it has been reported recently that $\mathrm{Dscam}^{-/-}$mice survived to adulthood in a BALB/cByJ-predominant mixed background (Fuerst et al., 2008). We therefore investigated the Dscam-deficient mouse on C57BL/6 and BALB/c mixed background. We found that these $D$ scam ${ }^{-/-}$mice indeed survived to adulthood. The plethysmography analyses revealed that the Dscam $^{+/-}$and Dscam ${ }^{-1-}$ mice with C57BL/6-BALB/c mixed background also showed irregular respiratory pattern (Figs. $2 B-D, 8 A)$. However, those irregularities, especially for the frequency of apnea (Fig. 2D), are milder compared with the C57BL/6 strain. The patch-clamp analyses revealed that the $\mathrm{C} 4$ activities of both $\mathrm{Dscam}^{+/-}$and $\mathrm{Dscam}^{-1-}$ mice on the C57BL/ $6-\mathrm{BALB} / \mathrm{c}$ mixed background showed slower frequencies and frequent apnea compared with that of Dscam wild-type, although these defects in $\mathrm{Dscam}^{-/-}$mice are milder than those on C57BL/6 background (Figs. $3 B, C, 8 B$ ). The defect of ventilatory response to hypercapnia in $\mathrm{Dscam}^{-1-}$ and $\mathrm{Dscam}^{+/-}$mice is not very much apparent on the $\mathrm{C} 57 \mathrm{BL} / 6-\mathrm{BALB} / \mathrm{c}$ mixed background
(Fig. 4C). Also very interestingly, the Pre-I neuronal activity is essentially maintained in Dscam ${ }^{-/-}$mice with C57BL/6 and $\mathrm{BALB} / \mathrm{c}$ mixed background (Fig. 9). These genetic background-dependent differences well correlated with the lethality.

\section{Discussion}

\section{DSCAM mediates synchronous activation of RVLM}

Pre-I neurons

In the present study, the results of both optical imaging and whole-cell patch-clamp analyses showed that the unilateral and bilateral synchrony of Pre-I neurons was reduced in $\mathrm{Dscam}^{+/-}$ mouse and lost in $\mathrm{Dscam}^{-1-}$ mouse, whereas that of Insp neurons in $\mathrm{Dscam}^{+/-}$and $\mathrm{Dscam}{ }^{-/-}$mice was retained, at least when triggered by $\mathrm{C} 4$ activities. How does DSCAM protein produce the synchronous Pre-I neuron activity? The Pre-I neurons in the pFRG may connect each other, relay their activities and synchronize, and then transfer them to the Insp neurons. For the connection of Pre-I neurons, DSCAM molecule may play critical roles. The neurite outgrowth assay of cultured DRG neurons in our present study indicated that the homophilic binding of DSCAM protein accelerates neurite extension. As described in the Introduction, a number of studies showed that DSCAM plays important roles in neural network formation. Although it is not yet known whether DSCAM in RVLM works through homophilic binding, resulting in either attraction or repulsion, these obser- 

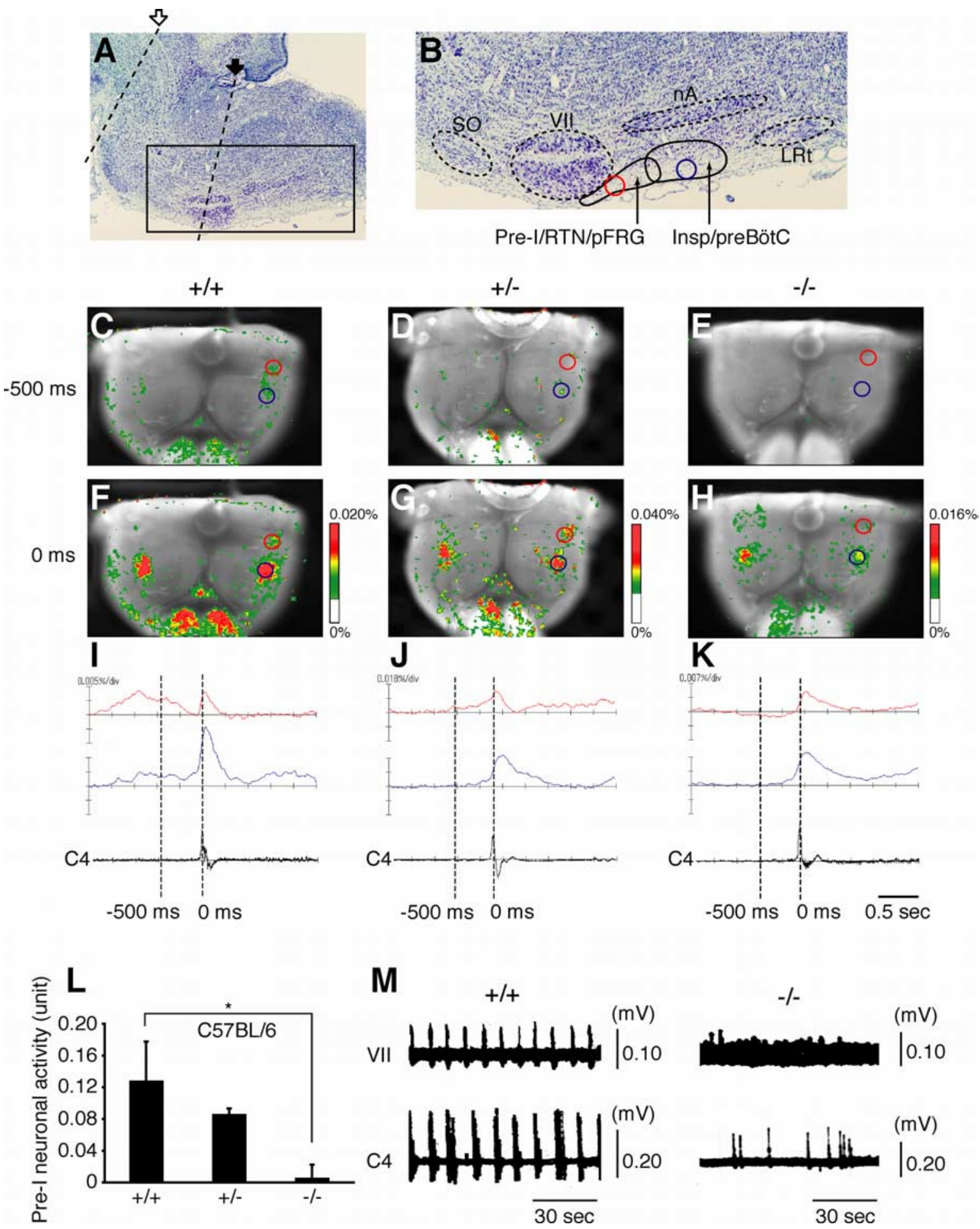

Figure 5. Defects of respiratory rhythm generation in medulla Pre-I neurons of Dscam-deficient mice (C57BL/ 6 background). $\boldsymbol{A}$, Parasagittal section of mouse brainstem at E19 stained by Nissl. A dashed line (filled arrow) indicates the anterior limit of the medulla-spinal cord preparation used for the optical imaging $(\boldsymbol{C}-\boldsymbol{K})$. Another dashed line (open arrow) indicates that used for the measurement of facial (VII) nerve activity $(\boldsymbol{M})$. $\boldsymbol{B}$, A magnified image of the squared region in $\boldsymbol{A}$. A red circle indicates the position of Pre-I neuronal activity, and a blue circle indicates that of Insp neuronal activity, which are shown in $\boldsymbol{C}-\boldsymbol{H}$. VII, Facial nucleus; LRt, lateral reticular nucleus; $\mathrm{nA}$, nucleus ambiguus; RTN, retrotrapezoid nucleus; $\mathrm{SO}$, superior olive. $\boldsymbol{C}-\boldsymbol{K}$, The $\Delta \boldsymbol{F} / \boldsymbol{F}$ pseudocolor images recorded $500 \mathrm{~ms}(\boldsymbol{C}-\boldsymbol{E})$ or $0 \mathrm{~ms}(\boldsymbol{F}-\boldsymbol{H})$ before $(4$ inspiratory burst are superimposed on the ventral surface of the medulla. Results of optical imaging are the average of 50 respiratory cycles triggered by 44 inspiratory activity. The red circles indicate the most prominent area of Pre-I neuronal activity, and the blue circles indicate that of Insp neuronal activity. Optical responses within red and blue circles (78 pixels) are quantified and shown as red and blue traces, respectively $(\boldsymbol{I}-\boldsymbol{K})$. Five mice, analyzed for each genotype, showed the same results and a representative trace from one mouse was shown here. The trace at the bottom (black) shows the $(4$ ventral root inspiratory activity. Each time point of the images in $\boldsymbol{C} \boldsymbol{H}$ is shown as vertical dotted lines in the graph (I-K). Note that the Pre-I neuron activity in Dscam ${ }^{-1-}$ mice disappeared $(\boldsymbol{E}, \boldsymbol{K})$, whereas the activity is moderately retained in Dscam ${ }^{+/-}$mice $(\boldsymbol{D}, \boldsymbol{J})$. The preparation was stained with $33 \mu \mathrm{g} / \mathrm{ml} \mathrm{Di}-2-\mathrm{ANEPEQ}$ for $30 \mathrm{~min}$. The exposure time was $20 \mathrm{~ms}$. Horizontal bar, $0.5 \mathrm{~s}$. The differences of the vertical scales in $\boldsymbol{I}-\boldsymbol{K}$ inevitably derived from the variable conditions, including degrees of staining. $\boldsymbol{L}$, Areas of Pre-I neuronal activity phase on the traces $(I-K)$ in wild-type $(n=5), \mathrm{Dscam}^{+/-}(n=5)$, and Dscam ${ }^{-1-}(n=8)$ mice were calculated by NIH ImageJ 1.33 u software. Unit in the ordinate represents the number of pixels that exists in the area. Area of Pre-I neuronal activity in $D$ scam ${ }^{-\prime-}$ mice was significantly smaller than that in wild-type mice. ${ }^{*} p<0.05$, Tukey-Kramer test compared with wild-type mice. Error bars represent SEM. M, The output of the VII nerve and the C 4 ventral root activities in medulla-spinal cord preparations of newborn mice. Horizontal bar under each activity represents a period of $30 \mathrm{~s}$. Vertical bar at the right side of VII nerve or C 4 activity represents an amplitude of 0.10 or $0.20 \mathrm{mV}$, respectively. The VII nerve activities for Dscam ${ }^{-/-}$became tonic. 


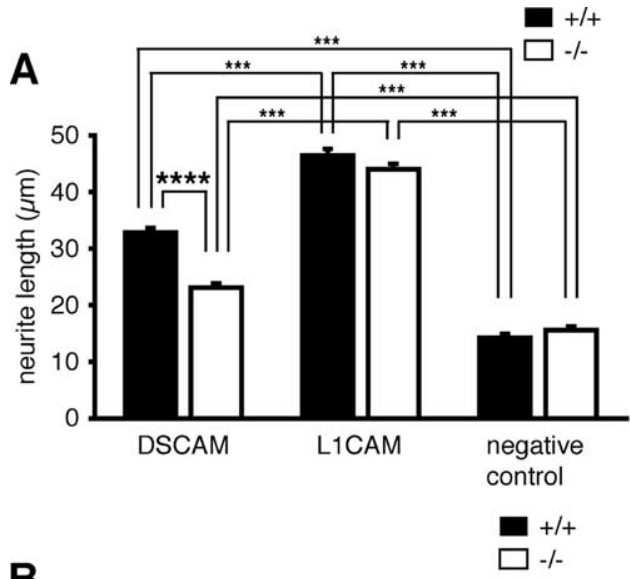

B

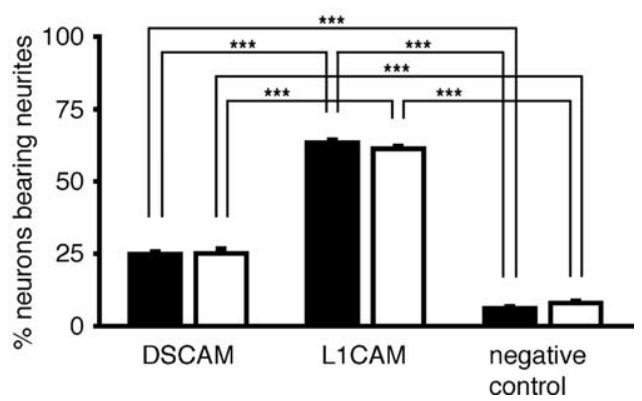

Figure 6. Dscam-dependent neurite outgrowth of DRG neurons. $A, B, D R G$ neurons isolated from wild-type or Dscam ${ }^{-1-}$ E19 mice (C57BL/6 background) were cultured for $7 \mathrm{~h}$ on the indicated substrates. The dishes were prepared by sequential coating with poly-D-lysine, anti-Fc antibody, and DSCAM-Fc or L1CAM-Fc chimeric proteins. Dishes coated with poly-D-lysine followed by anti-Fc antibody only were used as negative controls. $A$, The neurite length of wild-type or Dscam ${ }^{-1-}$ DRG neurons cultured on DSCAM ( $n=465$ or $\left.n=348\right)$, LICAM ( $n=$ 327 or $n=335)$, or without Fc-fusion protein (negative control) $(n=151$ or $n=142)$ was measured. The neurite length of DRG neurons on DSCAM-coated dish was significantly longer compared with that on negative control dish. ${ }^{* *} p<0.001$, Tukey-Kramer test compared with DRG neurons on anti-Fc antibody-coated dish. The neurite length of Dscam ${ }^{-1-}$ DRG neurons on a DSCAM-coated dish was significantly shorter than that of wild-type DRG neurons. ${ }^{* * * *} p<0.0001$, Student's $t$ test compared with wild-type neurons under the same culture conditions. $\boldsymbol{B}$, The percentage of neurons bearing neurites was quantified. Each bar represents six independent experiments. One hundred neurons were measured for each experiment. The percentage of DRG neurons bearing neurites did not differ significantly between Dscam ${ }^{-1-}$ and wild-type DRG neurons. Statistics on culture conditions with three entities (DSCAM-, L1CAM-, and without Fc-fusion protein-coated dishes) were calculated by Tukey-Kramer test. ${ }^{* * *} p<0.001$. Error bars represent SEM.

vations suggest that DSCAM in RVLM also plays critical roles in the formation of its integral neural network, and its deficiency in $\mathrm{Dscam}^{-1-}$ mice would lead to abnormal interconnections of Pre-I neurons and disruption of the synchroneity for Pre-I neurons as a whole.

We also found that the SMI311-positive neurons in the area dorsolateral to the facial nucleus were increased in Dscam $^{-1-}$ mice. This area locates dorsorostral to that of Pre-I neurons (Onimaru et al., 2006), and the increase in number of SMI311-positive neurons may possibly preclude the normal development of Pre-I neurons or inhibit the neuronal networks that would originally be generated. The reason for the neuronal increase is so far unknown, but this observation is reminiscent of the abnormal survival of neurons in mice deficient for the axon guidance molecule ephrin-A (Depaepe et al., 2005). Dscam may possibly have a similar cell-death receptor activity, as also suggested by decreased cell death in developing retina of the spontaneous Dscam mutant mouse (Fuerst et al., 2008).
Loss of Pre-I synchroneity in Dscam ${ }^{-/-}$mice causes abnormal central respiratory regulation and may result in perinatal lethality

The perinatal lethality observed in $\mathrm{Dscam}^{-1-}$ mouse may be attributable to abnormal central respiratory regulation caused by the loss of Pre-I neuron synchroneity and the defective ventilatory response to hypercapnia. So, why does the $\mathrm{Dscam}^{-1-}$ mouse show perinatal death and why does the $\mathrm{Dscam}^{+/-}$mouse can survive? The opiate surge at neonatal stage would depress the excitability of Insp neurons in the preBötC but not that of Pre-I neurons in the pFRG (Takeda et al., 2001). It is therefore hypothesized that the pFRG is an "anti-apnea" center that promotes breathing immediately after birth (Jacquin et al., 1996). Subsequently, the reorganization of the respiratory neuronal network, characterized by a reduction of pre-inspiratory activity in the pFRG, occurs at P1-P2 in rats (Oku et al., 2007). These reports suggest critical roles of Pre-I neurons at $\mathrm{P} 0$ and may explain the perinatal lethality of $D s c a m^{-1-}$ mouse. The retained Pre-I neuronal synchroneity in Dscam ${ }^{-1-}$ mouse on the C57BL/6 and $\mathrm{BALB} / \mathrm{c}$ mixed background that survived through the neonatal stage further suggest that the loss of Pre-I synchroneity surely causes the perinatal lethality. The moderate defect of Pre-I neurons in $\mathrm{Dscam}^{+/-}$mice may not be enough to kill the mice and let them survive the critical newborn period and grow mostly normally thereafter.

The loss of Pre-I neuron synchroneity would be the major defect in $\mathrm{Dscam}^{-1-}$ mouse, but it may not be the only one that explains the whole respiratory abnormalities in the mouse. Our results showed that naloxone, which can rescue the neonatal lethality of Krox $20^{-/-}$mice with defective Pre-I neurons by antagonizing the opiate surge (Jacquin et al., 1996), could not suppress the Dscam $^{-1-}$ phenotype. These results may indicate that Dscam $^{-1-}$ mouse also has critical abnormalities in Insp neurons in addition to Pre-I neurons. Although the activities of Insp neurons are synchronous to each other, the $\mathrm{C} 4$ activities synchronous to that of Insp neurons are irregular in $\mathrm{Dscam}^{+/-}$and $\mathrm{Dscam}^{-/-}$ mice, indicating that the rhythms of Insp neurons themselves are irregular in these mice. Furthermore, DSCAM is widely expressed in mouse nervous system, and therefore the possibilities of other unrecognized abnormalities contributing to the lethality may not be excluded. To understand the cellular mechanisms leading to these defects, additional investigations are required.

\section{Abnormalities in Dscam $^{-/-}$mouse are dosage dependent and sensitive to genetic backgrounds}

The phenotypic differences observed in $\mathrm{Dscam}^{-/-}$and $\mathrm{Dscam}^{+/-}$ mice indicate a dosage-dependent role of DSCAM in central respiratory regulation and are reminiscent of the dosagedependent natures of other neural Ig superfamily members, including neural cell adhesion molecule and neural cell adhesion L1-like (Stork et al., 1997; Frints et al., 2003). In addition, these DSCAM-dependent abnormalities are also highly genetic background dependent. The previously reported Dscam-deficient mouse, resulting from a spontaneous mutation, survived to adulthood under the BALB/CByJ-predominant background, whereas the mouse under the $\mathrm{C} 57 \mathrm{BL} / 6$ background died at neonatal stage (Fuerst et al., 2008). We confirmed that our Dscam ${ }^{-1-}$ mouse under 50\% C57BL/6 and 50\% BALB/c background also survived to adulthood. These observations suggest that the two strains, C57BL/6 and BALB/c, may have distinct alleles of genetic modifier(s) for the DSCAM-dependent respiratory function. Furthermore, we found that $\mathrm{Dscam}^{-1-}$ mice with 50\% C57BL/6 and 50\% 129 background, which was derived from our ES cells, 
A
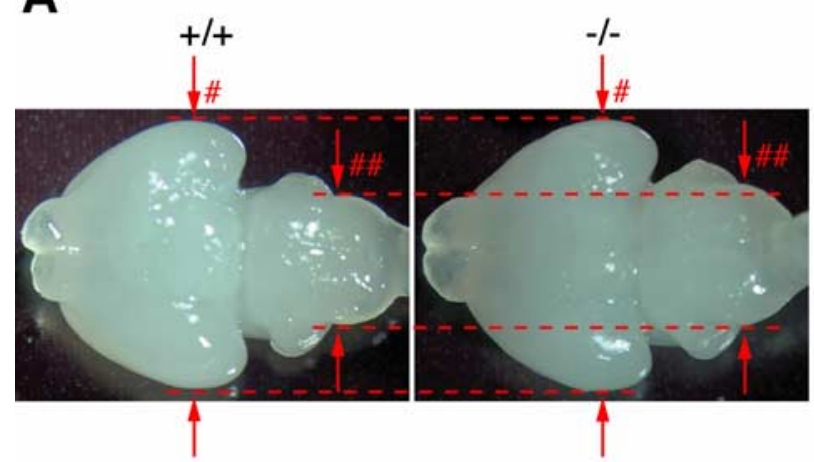

rostral
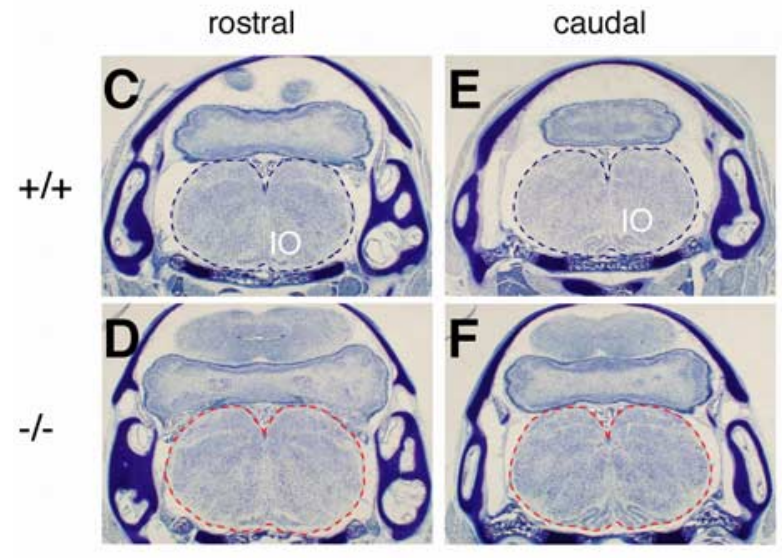

B

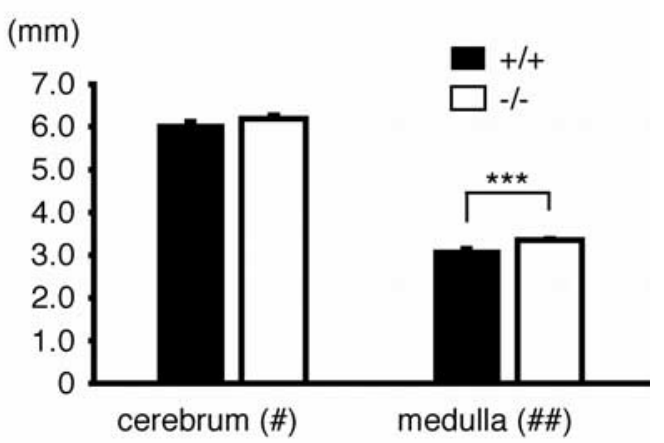

G

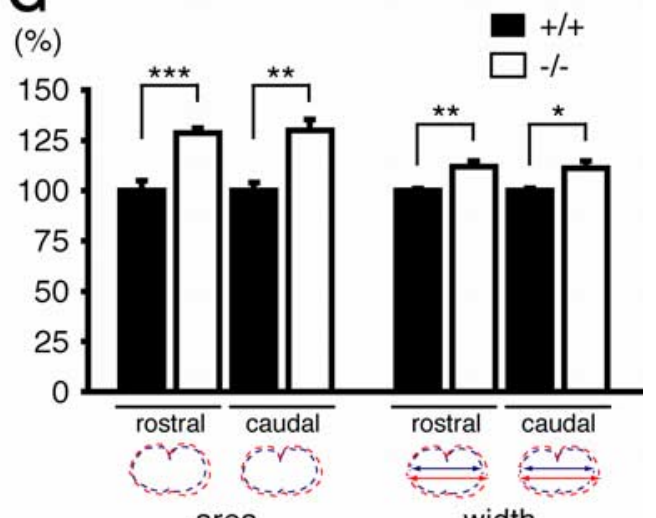

area

width
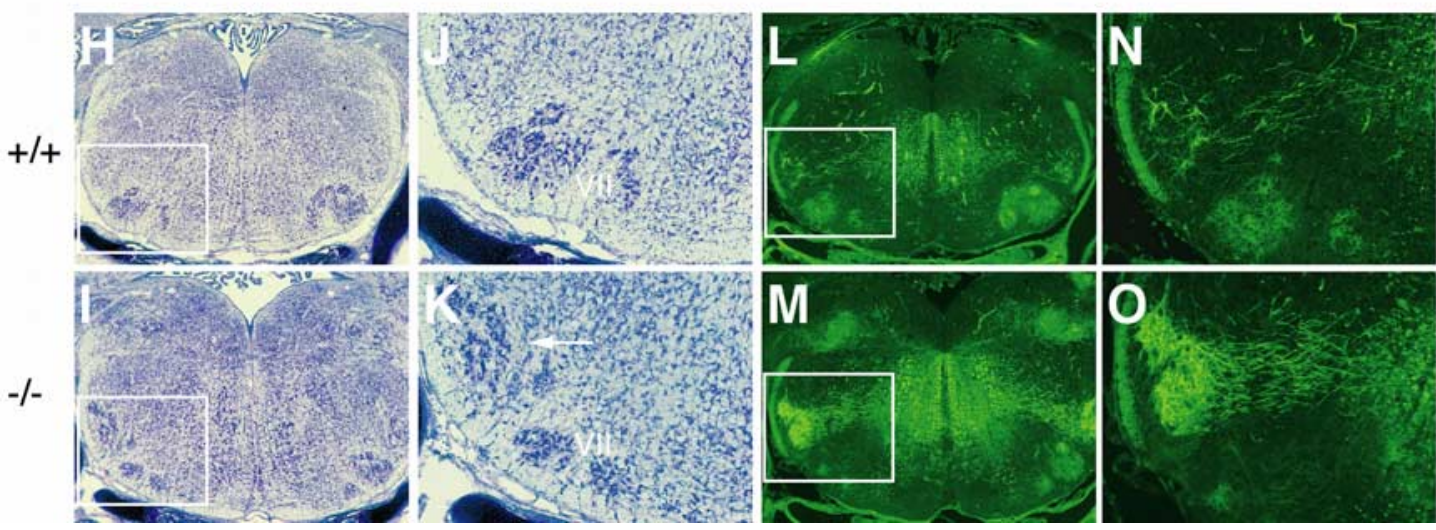

Figure 7. Enlargement of medulla in Dscam-deficient mouse neonates. A, Ventral views of wild-type and Dscam ${ }^{-1-}$ mouse brains at E19 (C57BL/6 background). Arrows indicate the sites for measurement of the width of cerebrum (\#) or medulla (\#\#). Dotted lines were provided for comparison. Note that the medulla of Dscam ${ }^{-1-}$ is larger than that of wild-type littermate. $B$, The averaged width of cerebrum (\#) and medulla (\#\#) are $6.05 \pm 0.07$ and $3.11 \pm 0.04 \mathrm{~mm}$, respectively, in wild-type $(n=7)$ and $6.20 \pm 0.07$ and $3.35 \pm 0.03 \mathrm{~mm}$, respectively, in Dscam ${ }^{-/-}$mice

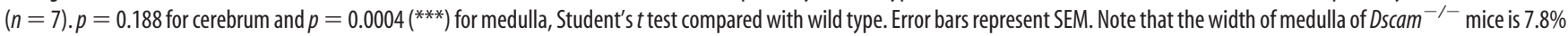
larger than that of wild-type littermates. $\mathbf{C} \boldsymbol{F}$, Coronal sections containing the rostral $(\boldsymbol{C}, \boldsymbol{D})$ or the caudal $(\boldsymbol{E}, \boldsymbol{F})$ part of medulla, including the inferior olive $(10)$ were stained by Nissl. Although the section for each genotype was prepared at identical anteroposterior positions (rostral end of an inferior olive and $\approx 150 \mu \mathrm{m}$ caudal to the rostral section), the sizes for the dorsal structures (cerebellum and inferior colliculus) look different because these were primarily affected by little changes of sectioning angles. The area of medulla (the area marked by a blue or a red dotted line) and the width of medulla (the length marked by a blue or a red arrow) in each of six wild-type or Dscam ${ }^{-1-}$ mice were measured and the values were standardized by wild-type as $100 \%$ (G). The medulla of Dscam ${ }^{-1-}$ mice $(\boldsymbol{D}, \boldsymbol{F})$ is larger in area than that of wild-type mice $(\boldsymbol{C}, \boldsymbol{E})$. The areas of medulla in Dscam ${ }^{-/-}$mice were significantly larger than that of wild-type littermates at the rostral $(28.7 \%$; $\left.{ }^{* * *} p=0.0004\right)$ and at the caudal $\left(30.0 \% ;{ }^{* *} p=0.0015\right)$ positions. The widths for those were also increased at the rostral $(12.0 \% ; * * 0.0021)$ and at the caudal $\left(11.2 \%\right.$; $\left.{ }^{*} p=0.0132\right)$ positions. Student's $t$ test compared with wild-type, respectively $(\boldsymbol{G})$. The sizes of cells and their densities in medulla are similar in Dscam ${ }^{-/-}$and wild type, suggesting that the total cell number is increased in Dscam ${ }^{-/}$. $\boldsymbol{H}-\mathbf{O}$, Serial coronal sections from wild-type $(\boldsymbol{H}, \boldsymbol{L})$ and $D_{\text {scam }}{ }^{--}(\boldsymbol{I}, \boldsymbol{M})$ mice at E19 were stained with Nissl $(\boldsymbol{H}-\boldsymbol{K})$ and SMI311 antibody recognizing nonphosphorylated neurofilaments, a marker for neuronal soma and dendrites $(\boldsymbol{L}-\mathbf{0})$. Magnified views of boxed areas in $\boldsymbol{H}, \boldsymbol{I}, \boldsymbol{L}$, or $\boldsymbol{M}$ were represented in $\boldsymbol{J}, \boldsymbol{K}, \boldsymbol{N}$, or $\boldsymbol{O}$, respectively. Increased number of Nissl-stained cells in the area dorsolateral to the VII nucleus was indicated by an arrow $(\boldsymbol{K})$. The numbers of SMI311-immunoreactive cells and fibers are increased in Dscam ${ }^{-/-}$mice $(\boldsymbol{M}, \mathbf{0})$.

died at perinatal stages. This may suggest that C57BL/6 and 129 trains have similar or identical alleles for the modifier(s). Interestingly, genetic background-dependent differences in phenotypes have also been reported in other neural Ig superfamily members. L1 cell adhesion molecule (L1)-deficient mice showed severe embryonic lethality under the C57BL/6 background, whereas the L1 knock-out mice survived to adulthood under the 129/Sv background (Dahme et al., 1997; Itoh et al., 2004). These 
observations may suggest that the high dosage dependency and the sensitiveness to genetic modifiers are common features to phenotypes caused by defects in the Ig superfamily members.

\section{$D S C A M$ as a candidate gene for central respiratory disorders}

The results reported here propose DSCAM as a promising candidate gene for human diseases with respiratory dysfunction, including congenital central hypoventilation syndrome (CCHS) and sudden infant death syndrome (SIDS). CCHS, also called Ondine's Curse disease, is a rare neonatal disorder characterized by abnormal ventilatory response to hypoxia and hypercapnia resulting from failure of autonomic respiratory control. Most CCHS patients show heterozygous de novo mutations in the PHOX2B gene (Amiel et al., 2003). Mutations in DSCAM could play modifying roles in CCHS or could be responsible for atypical CCHS cases.

Expression of Phox $2 b$ throughout the developing sympathetic, parasympathetic, and enteric ganglia suggested the involvement of PHOX2B in the establishment of the autonomic nervous system (Tiveron et al., 1996; Pattyn et al., 1997). Phox $2 b^{-/-}$ mouse embryos died in utero with absence of neurons or neuronal precursors at all sites of formation of autonomic ganglia (Pattyn et al., 1999). Observations of severely depressed respiratory stimulation by hypercapnia in patients with CCHS denote a failure of mechanisms that integrate chemoreceptor inputs to the respiratory centers (Spengler et al., 2001). Notably, PHOX2B is expressed in neurons that are a group of central chemoreceptor neurons in the retrotrapezoid nucleus as well as in a connected chain of neurons that relay information from peripheral chemoreceptors to the central respiratory column (Stornetta et al., 2006). The ventilatory response to hypercapnia in Phox $2 b^{+/-}$mice at P2 was approximately half of that in wild-type mice, because of their insufficient increase in respiratory frequency (Dauger et al., 2003). We also observed low ventilatory response to hypercapnia in Dscam $^{-1-}$ mice at $\mathrm{P} 0$, although the response in the heterozygote $\left(\right.$ Dscam $\left.^{+/-}\right)$was not significantly different from that in wild-type mice. Interestingly, the respiratory frequency under hypercapnia became slower than pre-hypercapnia in $\mathrm{Dscam}^{-1-}$ mice, whereas it became faster in Phox $2 b^{+/-}$mice, although it was still less than that of wild-type. The respiratory rhythm under normoxia was normal in Phox $2 b^{+/-}$mice (Dauger et al., 2003), which would be consistent with the fact that PHOX2B in adult rats was not expressed in the central respiratory pattern generator neurons themselves (Stornetta et al., 2006). The abnormalities observed in Pho $2 b^{+/-}$mice may originate from defects in a chemosensory circuit rather than the central respiratory pattern generator. In
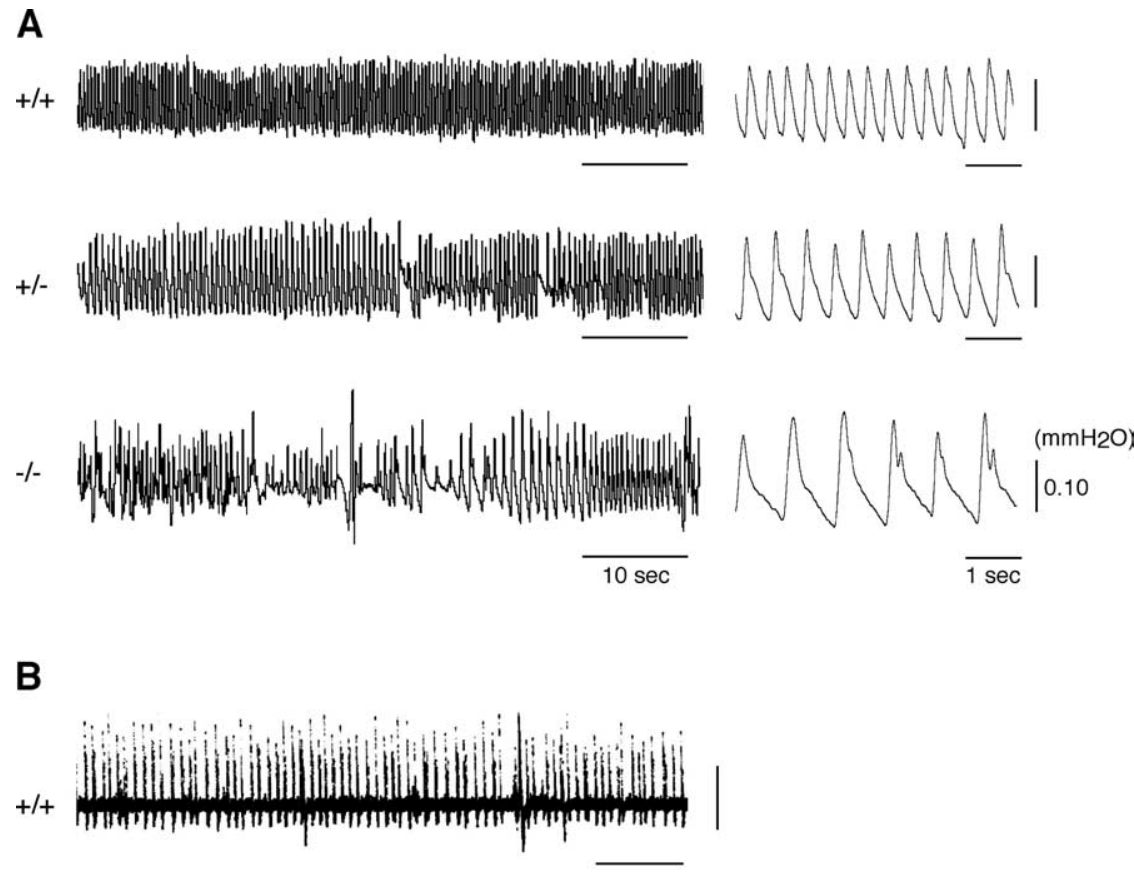

ure 8. Abnormal respiration in Dscam-deficient mice on C57BL/6-BALB/C mixed background. $A$, Spontaneous respiration activity of newborn (P0) mice was measured by plethysmography. Recordings correspond to spontaneous respiration in wildtype, Dscam ${ }^{+/-}$, and Dscam ${ }^{-/-}$littermates, respectively. Horizontal bar under each respiration activity represents a period of inspiratory activities in medulla-spinal cord preparations of newborn mice on the mixed background. Horizontal bar under each activities for Dscam ${ }^{-1-}$ mice were slow. Notably, low-amplitude (4 activity representing apnea was rarely observed in $D_{\text {scam }}{ }^{-1-}$ mice. For quantifications, see Figure 3.

contrast, the respiratory rhythm under normoxia is irregular in Dscam $^{-/-}$or even in Dscam ${ }^{+/-}$mice. Compared with the phenotypes of Phox $2 b^{+/-}$mice, the features of irregular respiration under normoxia and even slower respiratory frequency in response to hypercapnia are rather specific for $\mathrm{Dscam}^{-1-}$ (or $D_{\text {scam }}{ }^{+/-}$) mice. We previously reported that Dscam was expressed broadly in the brain, including the medulla and most or all of the peripheral ganglia (Yamakawa et al., 1998). Pre-I neurons in medulla have been reported to modulate the respiratory rhythm in response to $\mathrm{CO}_{2}$ changes in addition to the rhythm generator function (Kawai et al., 2006). This may suggest that the abnormal respiration in $\mathrm{Dscam}^{-/-}\left(\right.$or $\left._{\mathrm{Dscam}}{ }^{+/-}\right)$derives from defects in both chemosensation and rhythm generation. It remains as an important step to investigate whether DSCAM is 


\section{Dscam ${ }^{-/}$on C57BL/6-BALB/c mixed background}
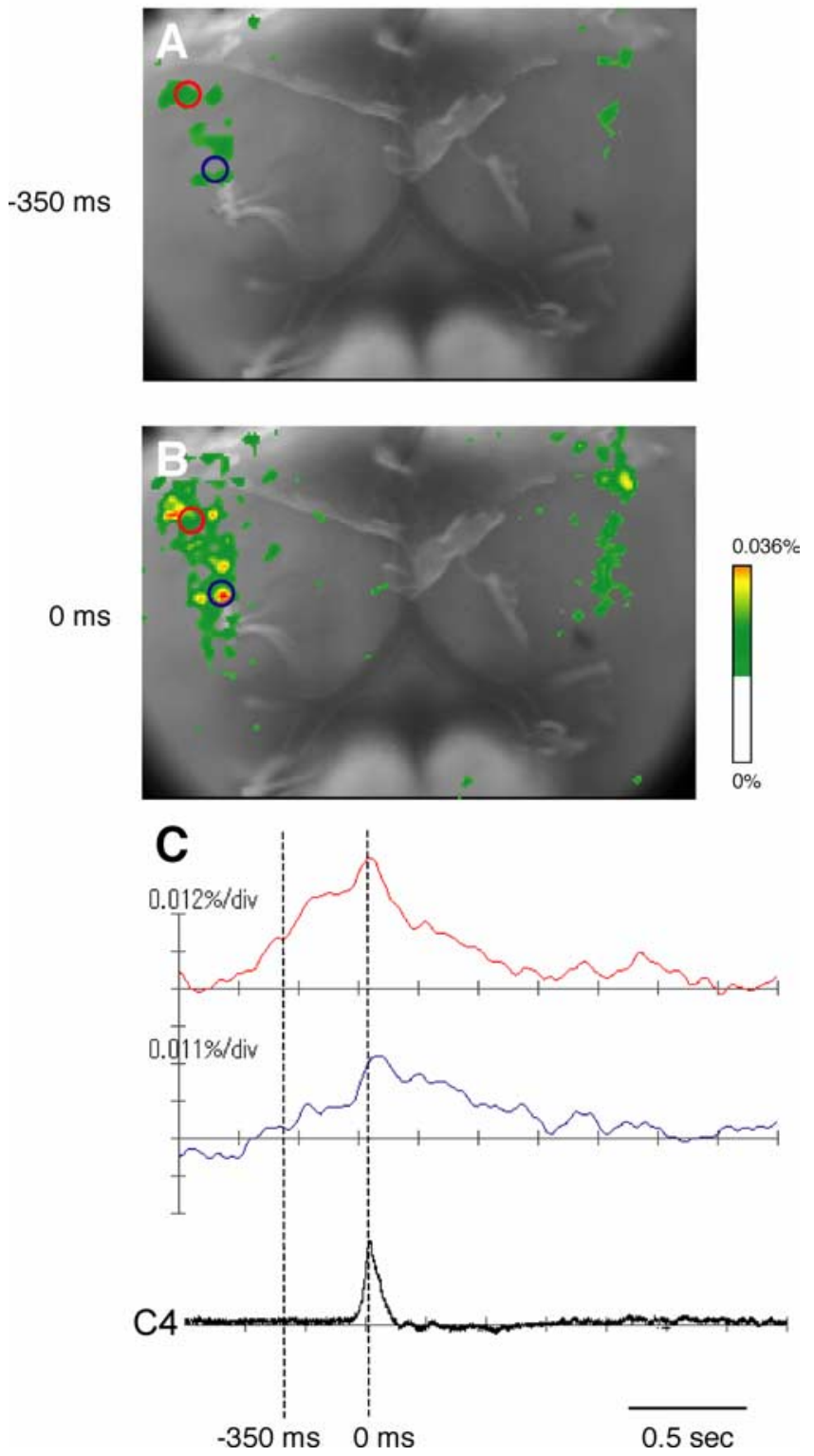

Figure 9. Retained Pre-I neuronal activity in Dscam-deficient mice on C57BL/6-BALB/C mixed background. The $\Delta F / F$ pseudocolor images on $-350 \mathrm{~ms}(\boldsymbol{A})$ or $0 \mathrm{~ms}(\boldsymbol{B})$ from C 4 inspiratory burst are superimposed on the ventral surface of the medulla. Results are the average of 50 respiratory cycles triggered by $(4$ inspiratory activity. The red circles indicate the most prominent area of Pre-I neuronal activity, and the blue circles indicate that of Insp neuronal activity. Optical responses within red and blue circles (78 pixels) are quantified and shown as red and blue traces, respectively (C). Two Dscam ${ }^{-/-}$mice showed the same results, and a representative trace from one mouse was shown here. The trace at the bottom (black) shows the (4 ventral root inspiratory activity. Note that the Pre-I neuron activity in Dscam ${ }^{-/-}$mice on C57BL/6 and $B A L B / C$ mixed background is essentially retained. The preparation was stained with $33 \mu \mathrm{g} / \mathrm{ml}$ Di-2-ANEPEQ for $30 \mathrm{~min}$. The exposure time was $20 \mathrm{~ms}$. Horizontal bar, $0.5 \mathrm{~s}$.

expressed in the Pre-I neurons themselves. Analyses on Pre-I or Insp neuronal activity in Phox $2 b^{+/-}$mice would also be useful to clarify the physiological function of $\mathrm{PHOX} 2 \mathrm{~B}$ and to distinguish between the functional properties of PHOX2B and DSCAM.

The abnormal central respiratory regulation observed in mice with Dscam deficiency, which is most severe in homozygotes, makes DSCAM an interesting candidate gene for central respiratory disorders, including SIDS, CCHS, or even milder respiratory diseases. Furthermore, it would be plausible that the overexpres- sion of DSCAM on chromosome 21q22 could contribute to some of neurodevelopmental defects in patients with Down syndrome, such as their central nervous system-dependent respiratory defects (Ferri et al., 1997, 1998) and/or mental retardation itself. Additional studies are required to investigate these possibilities.

\section{References}

Agarwala KL, Nakamura S, Tsutsumi Y, Yamakawa K (2000) Down syndrome cell adhesion molecule DSCAM mediates homophilic intercellular adhesion. Brain Res Mol Brain Res 79:118-126.

Agarwala KL, Ganesh S, Amano K, Suzuki T, Yamakawa K (2001) DSCAM, a highly conserved gene in mammals, expressed in differentiating mouse brain. Biochem Biophys Res Commun 281:697-705.

Amiel J, Laudier B, Attié-Bitach T, Trang H, de Pontual L, Gener B, Trochet D, Etchevers H, Ray P, Simonneau M, Vekemans M, Munnich A, Gaultier C, Lyonnet S (2003) Polyalanine expansion and frameshift mutations of the paired-like homeobox gene $P H O X 2 B$ in congenital central hypoventilation syndrome. Nat Genet 33:459-461.

Arata A, Onimaru H, Homma I (1990) Respiration-related neurons in the ventral medulla of newborn rats in vitro. Brain Res Bull 24:599-604.

Barlow GM, Micales B, Chen XN, Lyons GE, Korenberg JR (2002) Mammalian DSCAMs: roles in the development of the spinal cord, cortex, and cerebellum? Biochem Biophys Res Commun 293:881-891.

Dahme M, Bartsch U, Martini R, Anliker B, Schachner M, Mantei N (1997) Disruption of the mouse $L 1$ gene leads to malformations of the nervous system. Nat Genet 17:346-349.

Dauger S, Pattyn A, Lofaso F, Gaultier C, Goridis C, Gallego J, Brunet JF (2003) Phox 2 b controls the development of peripheral chemoreceptors and afferent visceral pathways. Development 130:6635-6642.

Depaepe V, Suarez-Gonzalez N, Dufour A, Passante L, Gorski JA, Jones KR, Ledent C, Vanderhaeghen P (2005) Ephrin signalling controls brain size by regulating apoptosis of neural progenitors. Nature 435:1244-1250.

Feldman JL, Mitchell GS, Nattie EE (2003) Breathing: rhythmicity, plasticity, chemosensitivity. Annu Rev Neurosci 26:239-266.

Ferri R, Curzi-Dascalova L, Del Gracco S, Elia M, Musumeci SA, Stefanini MC (1997) Respiratory patterns during sleep in Down's syndrome: importance of central apnoeas. J Sleep Res 6:134-141.

Ferri R, Curzi-Dascalova L, Del Gracco S, Elia M, Musumeci SA, Pettinato S (1998) Heart rate variability and apnea during sleep in Down's syndrome. J Sleep Res 7:282-287.

Fransen E, D’Hooge R, Van Camp G, Verhoye M, Sijbers J, Reyniers E, Soriano P, Kamiguchi H, Willemsen R, Koekkoek SK, De Zeeuw CI, De Deyn PP, Van der Linden A, Lemmon V, Kooy RF, Willems PJ (1998) L1 knockout mice show dilated ventricles, vermis hypoplasia and impaired exploration patterns. Hum Mol Genet 7:999-1009.

Frints SG, Marynen P, Hartmann D, Fryns JP, Steyaert J, Schachner M, Rolf B, Craessaerts K, Snellinx A, Hollanders K, D’Hooge R, De Deyn PP, Froyen G (2003) CALL interrupted in a patient with non-specific mental retardation: gene dosage-dependent alteration of murine brain development and behavior. Hum Mol Genet 12:1463-1474.

Fuerst PG, Koizumi A, Masland RH, Burgess RW (2008) Neurite arborization and mosaic spacing in the mouse retina require DSCAM. Nature 451:470-474.

Fusaoka E, Inoue T, Mineta K, Agata K, Takeuchi K (2006) Structure and function of primitive immunoglobulin superfamily neural cell adhesion molecules: a lesson from studies on planarian. Genes Cells 11:541-555.

Hughes ME, Bortnick R, Tsubouchi A, Bäumer P, Kondo M, Uemura T, Schmucker D (2007) Homophilic Dscam interactions control complex dendrite morphogenesis. Neuron 54:417-427.

Hummel T, Vasconcelos ML, Clemens JC, Fishilevich Y, Vosshall LB, Zipursky SL (2003) Axonal targeting of olfactory receptor neurons in Drosophila is controlled by Dscam. Neuron 37:221-231.

Itoh K, Cheng L, Kamei Y, Fushiki S, Kamiguchi H, Gutwein P, Stoeck A, Arnold B, Altevogt P, Lemmon V (2004) Brain development in mice lacking L1-L1 homophilic adhesion. J Cell Biol 165:145-154.

Jacquin TD, Borday V, Schneider-Maunoury S, Topilko P, Ghilini G, Kato F, Charnay P, Champagnat J (1996) Reorganization of pontine rhythmogenic neuronal networks in Krox-20 knockout mice. Neuron 17:747-758

Kamiguchi H, Yoshihara F (2001) The role of endocytic 11 trafficking in polarized adhesion and migration of nerve growth cones. J Neurosci 21:9194-9203.

Kawai A, Onimaru H, Homma I (2006) Mechanisms of $\mathrm{CO}_{2} / \mathrm{H}^{+}$chemore- 
ception by respiratory rhythm generator neurons in the medulla from newborn rats in vitro. J Physiol 572:525-537.

Kuwaki T, Cao WH, Kurihara Y, Kurihara H, Ling GY, Onodera M, Ju KH, Yazaki Y, Kumada M (1996) Impaired ventilatory responses to hypoxia and hypercapnia in mutant mice deficient in endothelin-1. Am J Physiol 270:R1279-R1286.

Ly A, Nikolaev A, Suresh G, Zheng Y, Tessier-Lavigne M, Stein E (2008) DSCAM is a netrin receptor that collaborates with DCC in mediating turning responses to netrin-1. Cell 133:1241-1254.

Matthews BJ, Kim ME, Flanagan JJ, Hattori D, Clemens JC, Zipursky SL, Grueber WB (2007) Dendrite self-avoidance is controlled by Dscam. Cell 129:593-604.

Nakata A, Kamiguchi H (2007) Serine phosphorylation by casein kinase II controls endocytic L1 trafficking and axon growth. J Neurosci Res $85: 723-734$

Oku Y, Masumiya H, Okada Y (2007) Postnatal developmental changes in activation profiles of the respiratory neuronal network in the rat ventral medulla. J Physiol 585:175-186.

Onimaru H, Homma I (1992) Whole cell recordings from respiratory neurons in the medulla of brainstem-spinal cord preparations isolated from newborn rats. Pflugers Arch 420:399-406.

Onimaru H, Homma I (2003) A novel functional neuron group for respiratory rhythm generation in the ventral medulla. J Neurosci 23:1478-1486.

Onimaru H, Arata A, Arata S, Shirasawa S, Cleary ML (2004) In vitro visualization of respiratory neuron activity in the newborn mouse ventral medulla. Brain Res Dev Brain Res 153:275-279.

Onimaru H, Kumagawa Y, Homma I (2006) Respiration-related rhythmic activity in the rostral medulla of newborn rats. J Neurophysiol 96:55-61.

Paton JFR (1996) A working heart-brainstem preparation of the mouse. J Neurosci Methods 65:63-68.

Pattyn A, Morin X, Cremer H, Goridis C, Brunet JF (1997) Expression and interactions of the two closely related homeobox genes Phox $2 a$ and Phox $2 b$ during neurogenesis. Development 124:4065-4075.

Pattyn A, Morin X, Cremer H, Goridis C, Brunet JF (1999) The homeobox gene Phox $2 b$ is essential for the development of autonomic neural crest derivatives. Nature 399:366-370.

Rekling JC, Feldman JL (1998) PreBötzinger complex and pacemaker neurons: hypothesized site and kernel for respiratory rhythm generation. Annu Rev Physiol 60:385-405.

Sakai K, Miyazaki J (1997) A transgenic mouse line that retains Cre recombinase activity in mature oocytes irrespective of the cre transgene transmission. Biochem Biophys Res Commun 237:318-324.
Schmucker D, Clemens JC, Shu H, Worby CA, Xiao J, Muda M, Dixon JE, Zipursky SL (2000) Drosophila Dscam is an axon guidance receptor exhibiting extraordinary molecular diversity. Cell 101:671-684.

Shirasawa S, Arata A, Onimaru H, Roth KA, Brown GA, Horning S, Arata S, Okumura K, Sasazuki T, Korsmeyer SJ (2000) Rnx deficiency results in congenital central hypoventilation. Nat Genet 24:287-290.

Smith JC, Ellenberger HH, Ballanyi K, Richter DW, Feldman JL (1991) PreBötzinger complex: a brainstem region that may generate respiratory rhythm in mammals. Science 254:726-729.

Soba P, Zhu S, Emoto K, Younger S, Yang SJ, Yu HH, Lee T, Jan LY, Jan YN (2007) Drosophila sensory neurons require Dscam for dendritic selfavoidance and proper dendritic field organization. Neuron 54:403-416.

Spengler CM, Gozal D, Shea SA (2001) Chemoreceptive mechanisms elucidated by studies of congenital central hypoventilation syndrome. Respir Physiol 129:247-255.

Stork O, Welzl H, Cremer H, Schachner M (1997) Increased intermale aggression and neuroendocrine response in mice deficient for the neural cell adhesion molecule (NCAM). Eur J Neurosci 9:1117-1125.

Stornetta RL, Moreira TS, Takakura AC, Kang BJ, Chang DA, West GH, Brunet JF, Mulkey DK, Bayliss DA, Guyenet PG (2006) Expression of Phox $2 \mathrm{~b}$ by brainstem neurons involved in chemosensory integration in the adult rat. J Neurosci 26:10305-10314.

Takeda S, Eriksson LI, Yamamoto Y, Joensen H, Onimaru H, Lindahl SGE (2001) Opioid action on respiratory neuron activity of the isolated respiratory network in newborn rats. Anesthesiology 95:740-749.

Tiveron MC, Hirsch MR, Brunet JF (1996) The expression pattern of the transcription factor Phox2 delineates synaptic pathways of the autonomic nervous system. J Neurosci 16:7649-7660.

Wang J, Zugates CT, Liang IH, Lee CH, Lee T (2002) Drosophila Dscam is required for divergent segregation of sister branches and suppresses ectopic bifurcation of axons. Neuron 33:559-571.

Yamagata M, Sanes JR (2008) Dscam and Sidekick proteins direct laminaspecific synaptic connections in vertebrate retina. Nature 451:465-469.

Yamakawa K, Huot YK, Haendelt MA, Hubert R, Chen XN, Lyons GE, Korenberg JR (1998) DSCAM: a novel member of the immunoglobulin superfamily maps in a Down syndrome region and is involved in the development of the nervous system. Hum Mol Genet 7:227-237.

Yimlamai D, Konnikova L, Moss LG, Jay DG (2005) The zebrafish down syndrome cell adhesion molecule is involved in cell movement during embryogenesis. Dev Biol 279:44-57. 